\title{
Detection of structures on multiple scales around the South Galactic Pole.
}

\author{
A two-dimensional analysis of the COSMOS/UKST Southern Sky Galaxy Catalogue
}

\author{
E. Escalera and H.T. MacGillivray \\ Royal Observatory, Blackford Hill, Edinburgh, EH9 3HJ, UK
}

Received June 26; accepted December 14, 1995

\begin{abstract}
We report the results of a 2D analysis of distributions of galaxies in a search for structures on a wide range of scales, from clusters up to superclusters and beyond, including the identification of voids. The structures are identified in data from the COSMOS/UKST Southern Sky Galaxy Catalogue (CUSSGC). The subsample used in this first investigation contains some $1.410^{6}$ galaxies in an area of $\sim 69 \times 44$ degrees around the South Galactic Pole. From our analysis, we find 25 very large-scale structures (of 10 degrees or more in extent), about 60 structures of intermediate scales (superclusters and/or elongated features), and more than 220 cluster-sized objects. All these features are significant, having high confidence levels for detection. Some extended voids are also detected in this area, likewise with high significance. This work is based on the use of the wavelet transform, the method of which has already been extensively described in a previous paper in which we demonstrated the principle of the technique. The wavelet analysis gives the position of the structures detected, together with significance levels and some parametrisation of their properties (extent, population, elongation, etc). Ultimately, our aim is the construction of a large and homogeneous database of structures on all scales in this important part of the southern sky. ${ }^{\star}$
\end{abstract}

Key words: cosmology: large-scale structure of Universe — galaxies: clusters — surveys — methods: data analysis

\section{Introduction}

This work tackles the controversial question of the spatial distribution of galaxies in the Universe, on several scales. The observed distribution, in particular the frequency and properties of large scale vs. small scale structures, should in principle help to discriminate between competing models for the formation and evolution of the matter.

In a recent paper (Escalera \& MacGillivray 1995, hereafter EMG, and references contained therein) we described the main aspects of the subject. Although the present paper is a direct continuation of EMG, we first briefly recall the salient points of the topics concerned. For completeness, the essential references are repeated herein, although readers may refer to EMG for a more comprehensive list.

The topology observed today results from both the initial conditions and the subsequent mechanisms of formation of the structures. Even if these mechanisms are widely agreed to be gravitational collapses of initially Gaussian random fluctuations, the topology still depends

Send offprint requests to: Harvey T. MacGillivray

${ }^{\star}$ Tables 2 to 13 are only available in electronic form at the CDS via anonymous ftp 130.79.128.5 on what was the initial power spectrum. Many possible scenarios are combined and each resulting model predicts its particular topology. For example, good agreement is reached with the conclusion of Gott et al. (1989) who state that the large-scale structure of the Universe is spongy rather than bubbly. This supports the comfortable idea that both galaxies and large-scale structures formed from gravitational instability in an inflationary stage of the early evolution of the Universe. Other models however depart from gravitational instabilities. These are, for example, the relatively popular "explosive" scenarios (Ikeuchi 1981; Ostriker \& Cowie 1981), the "cosmic string" models (Vilenkin 1985, Brandenberger 1989), and others theorising even currently unknown physical processes (Messina et al. 1990; Moscardini et al. 1991). These models, but not exclusively, are consistent with Large-scale non-Gaussian features (structures and also voids). To date, a reliable "all-scales topology" of the Universe is absent, and so the debate remains open.

Extracting a wide-field, objective, multi-scale topology is perhaps the best way to proceed for the abovementioned purpose. As stated in EMG, our present contribution consists in combining a method and a dataset 
fully consistent with each other and both well adapted for progressing towards that particular goal. The method is based on the wavelet transform, which provides for the objective detection of multi-scale features. The data come from the COSMOS/UKST Southern Sky Galaxy Catalogue (MacGillivray \& Yentis 1994), which is a machineproduced catalogue obtained from objective automated procedures, in other words a photometric spatial distribution of galaxies around the South Galactic Pole.

In Sect. 2 we recall the essential characteristics (i.e. advantages and inconveniences) of the method, and this is repeated in Sect. 3 for the data. The main part of this work is contained in Sects. 4 and 5, which detail the results obtained for Large-scale and Small-scale investigations respectively. These latter two sections both include some explanation of the successive sampling of the catalogue, the main results (complete set of figures, tables of properties, and comments), and a discussion on the structures detected. Sect. 6 is devoted exclusively to the detection of voids, including full results and discussion. Sect. 7 is the core of this present paper, where the main database is given, all scales together, in the form of a unique table. In Sect. 8 we give a general discussion of the main results. Sect. 8.1 provides a comparison of our objects with the classical ACO catalogue. In Sect. 8.2 we provide a list of possible further applications of this work. Finally, in Sect. 9 we summarise the progress achieved towards our main aim.

\section{Topology with the wavelet transform}

A wavelet is a bound function, invariant in both translation and dilatation. As a result, the convolution of a wavelet with a given signal provides a local and multi-scale exploration of it, pointing out any event (discontinuity) it may include. The transform produces a set of wavelet coefficients, the so-called wavelet image. This is the spirit of the wavelet transform. The first introduction of wavelets to astrophysics was made by Slezak et al. (1989) who describe how it is successfully used to detect galaxy structures in a given field. The main details of the procedure we use are given also in our demonstrating paper (EMG), and it is beyond the scope of the present paper to review any general property of the method.

The results presented hereafter need, however, to retain some particular aspect. The crucial point is that a full set of successive scales are simultaneously investigated, with significance levels computed at each scale. Thus, a given structure is generally detected at several consecutive scales, and we only retain the one which produces the best contrast, hence the highest significance level. The value of such an optimal scale is closely related to the real dimension of the structure, in so far as the concept of finite dimension is valid for objects lying superimposed on continuous density fields. So, from our procedure each structure is represented by its corresponding optimal scale, i.e. the area responsible for that particular signal. Once detected, some characteristics are extracted from the wavelet images. The local maximum of the wavelet coefficients locates a given structure, while a particular isophote surrounding the maximum gives indications of the shape, size and orientation of the structure. In the following, such a specific isophote is fitted by an ellipse, leading thus to estimate the orientation of the structure, and its dimension and shape (semi-axes); finally the position of the centroid of the ellipse is an alternative indication of the position of the structure, which should not necessarily coincide with the location of the local maximum, except of course for those structures fully regular in their density profile.

Another fundamental aspect is the choice of a wavelet function, called "Mexican Hat", which is the second derivative of a Gaussian: the transform is thus insensitive to a constant gradient, and so structures of a given size are detected even if superimposed on the gradient of larger ones.

At this point in the process, we must recall that the data analysed consist of a spatial map of galaxies that naturally comes from the projection of a three-dimensional distribution. As pointed out by Coles \& Plionis (1991), the influence of projection effects is rather hard to determine analytically since we ignore (by definition) the pattern of the real thing. Such a three-dimensional distribution contains both "true" and random structures, and once projected the latter are in principle not easily distinguishable from the former. These inconveniences, however, are removed by the use of a systematic procedure: the significance levels are computed from Monte-Carlo simulations that mimic the characteristics of the real data (Escalera \& Mazure 1992). Such exact replicas contain all the expected random fluctuations (two-dimensional or projected-threedimensional), and so we are allowed to simply select from the real data those structures that are not reproduced by any random mechanism. Alternatively, the significance level $P_{\mathrm{SL}}$ accorded to the individual structures is derived from the simulations that contain a structure higher or equal to that observed in the real field.

\section{The data}

The motivation for performing the multi-scale analysis presented herein comes from the characteristics of the catalogue itself. The $C U S S G C$ is a two-dimensional map of the distribution of galaxies throughout an extended area of $\sim 2.5$ steradians around the South Galactic pole. It is $95 \%$ complete and homogeneous within the whole extension, down to the limiting magnitude of $B_{j}=20.5$. This is an opportunity to perform completely a multi-scale detection of structures, since both homogeneity and completeness are necessary conditions of the performance of the method used. Also, the extent is large enough to provide evidence of very large structures, a class of objects which is still ill-defined. The work presented here deals with a 
sample containing 1.417.233 galaxies. It represents about $60 \%$ of the total available area, covering some $8.5 \%$ of the southern sky within the following limits (corresponding to Equinox J2000):

- Right Ascension $=21 \mathrm{~h} 08 \mathrm{~m}$ to $1 \mathrm{~h} 44 \mathrm{~m}$

- Declination $=-60.0$ deg. to $-20.0 \mathrm{deg}$.

\section{Wide-field detection}

The search for large scale structures is a difficult task due to the extensive amount of data which has to be managed. We had to introduce a specific procedure in order to make things suitable, according to both practical requirements and theoretical interpretation. In our case, we subsampled the dataset in successive magnitude slices: i.e. the complete sample is divided into selected ranges of magnitudes. The advantages of this are multiple. On the one hand, this fulfills the technical constraints, since both the computation time and the number of galaxies managed become reasonable. On the other hand, it preempts the question of projection effects, which are the major problem for any $2 \mathrm{D}$ analysis. The magnitude slices should not contain "projected structures" if they are thin enough compared to the size of the features one wants to detect. And they still remain a fair tracer of large structures, simply by positioning them at regular distances, once again according to the scale investigated. In Table 1 we detail the characteristics of the 12 magnitude slices used, hereafter refered as "domains". In the following we will refer to these domains by codes as given in this table, i.e. [D085] to [D205].

The first three domains (down to $B_{j}=17.3$ ) are consecutive and put together they fulfill the whole corresponding space: excessive populations and projection effects are not dominant problems when considering only bright galaxies, and so it was decided to combine them. The following nine are true slices: the respective locations are selected from the main histogram (population vs. magnitude) in order to get them almost equally positioned in terms of true distance. This is done to avoid losing any structure between two successive slices; every large scale structure is crossed by at least one slice and hence will give its signature. Accordingly, depths in magnitudes are computed to correspond to almost identical depths in true dimensions, so each slice is equally representative of the local distribution. It must be noted that we do not expect to retrieve the whole of structures, but an unambiguous evidence of their presence and distribution (see Melott et al. 1989, for an extensive investigation of the behaviour of two-dimensional slices through three-dimensional distributions). Alternatively, our procedure also gives an estimate of their extent and orientation.

Figures 1 to 12 illustrate all the structures detected in the consecutive domains. For all of them we present the galaxy distribution (Figs. "A") and the corresponding map of the structures, represented by ellipses (Figs. "B").
Table 1. Definition of the subsamples ("domains") successively used for the detection. Column (1) gives the codes that will be used throughout this paper. Column (2) lists the number of galaxies $\left(N_{\text {gal }}\right)$ involved in the domains. Columns $(5)$ and (6) give the limits in the $B_{j}$ magnitude. Note that the first 3 subsamples are consecutive (the sum involves all the available data), while the remaining 9 are thinner and thinner magnitude slices. Column (7) is a parameter to take into account a distance effect (see text): $f_{i}$ is the rate in dimensions from one field to another (computation starts from [D180]). The extension is common to all the subsamples: right ascension $\alpha=-40$ to +29 degrees, and declination $\delta=-62$ to -18 degrees

\begin{tabular}{||l|c|c|c|c|}
\hline $\begin{array}{l}\text { Sample } \\
(1)\end{array}$ & $\begin{array}{c}N_{\text {gal }} \\
(2)\end{array}$ & $\begin{array}{c}M_{\text {inf }} \\
(3)\end{array}$ & $\begin{array}{c}M_{\text {sup }} \\
(4)\end{array}$ & $\begin{array}{c}f_{i} \\
(5)\end{array}$ \\
\hline D085 & 8697 & 8.54 & 16.43 & - \\
D164 & 8971 & 16.43 & 16.96 & - \\
D169 & 8907 & 16.96 & 17.26 & - \\
D180 & 8517 & 18.00 & 18.07 & 1.00 \\
D185 & 9316 & 18.50 & 18.53 & 1.21 \\
D190 & 7639 & 19.00 & 19.02 & 1.46 \\
D193 & 5168 & 19.33 & 19.34 & 1.64 \\
D196 & 6602 & 19.66 & 19.67 & 1.85 \\
D200 & 7662 & 20.00 & 20.01 & 2.13 \\
D201 & 8108 & 20.13 & 20.14 & 2.23 \\
D203 & 8297 & 20.25 & 20.26 & 2.35 \\
D205 & 8328 & 20.49 & 20.50 & 2.58 \\
\hline
\end{tabular}

These figures are not corrected for topological effects in order to allow them to be superposed; thus it must be noticed that the visual impressions can differ from the numerical parameters listed in the tables. Labels are applied to organise the many features detected, and no connection a priori must be seen between structures with same generic names (e.g. Ba, Bc), except to belong approximately to the same domain. When two capital letters are used, this indicates a coincidence in position within nonconsecutive domains, but again without any assumption as to the identity of the structures (e.g. AN located "behind" A). 


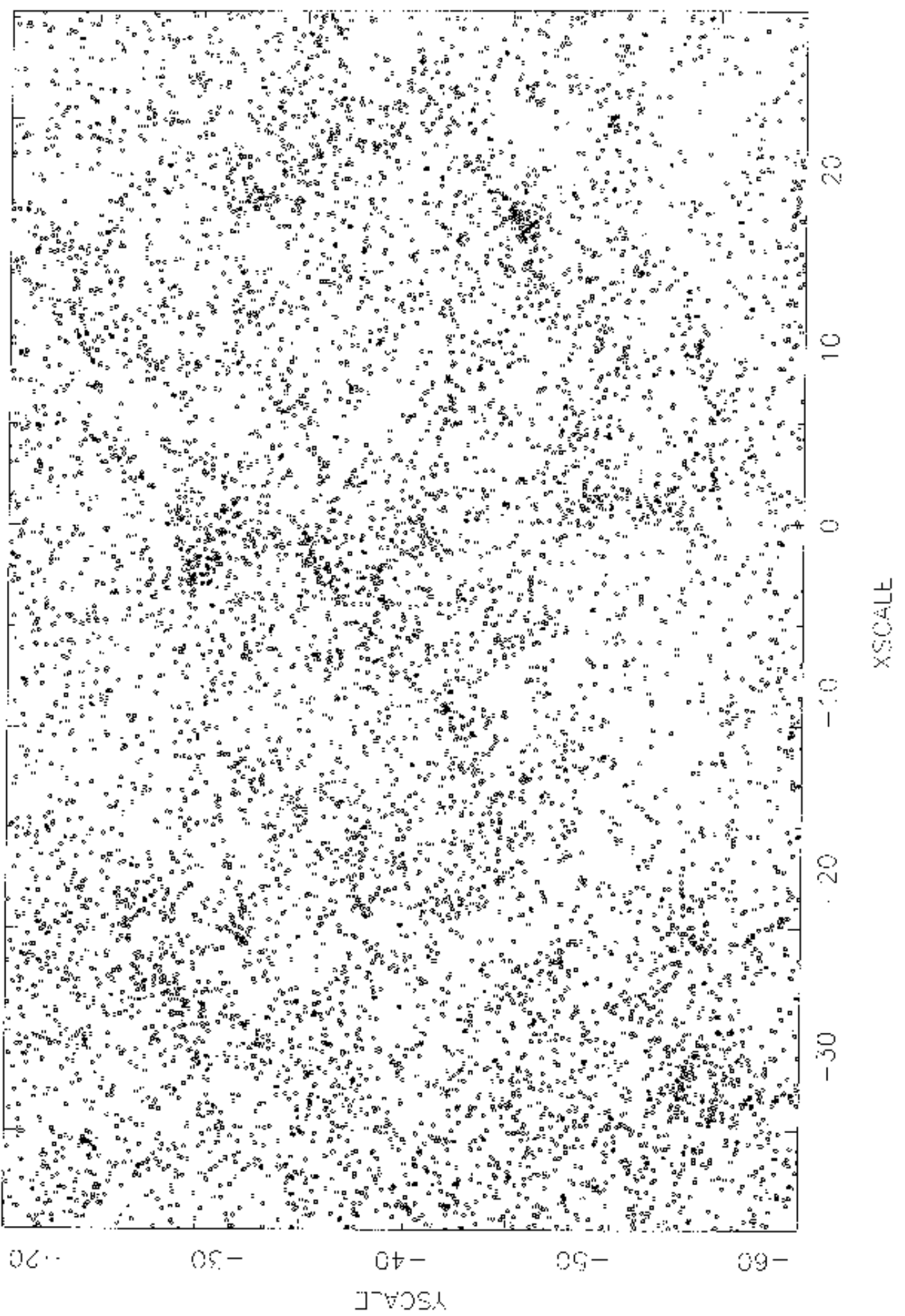

Fig. 1. a) Spatial distribution of galaxies in the domain D085 


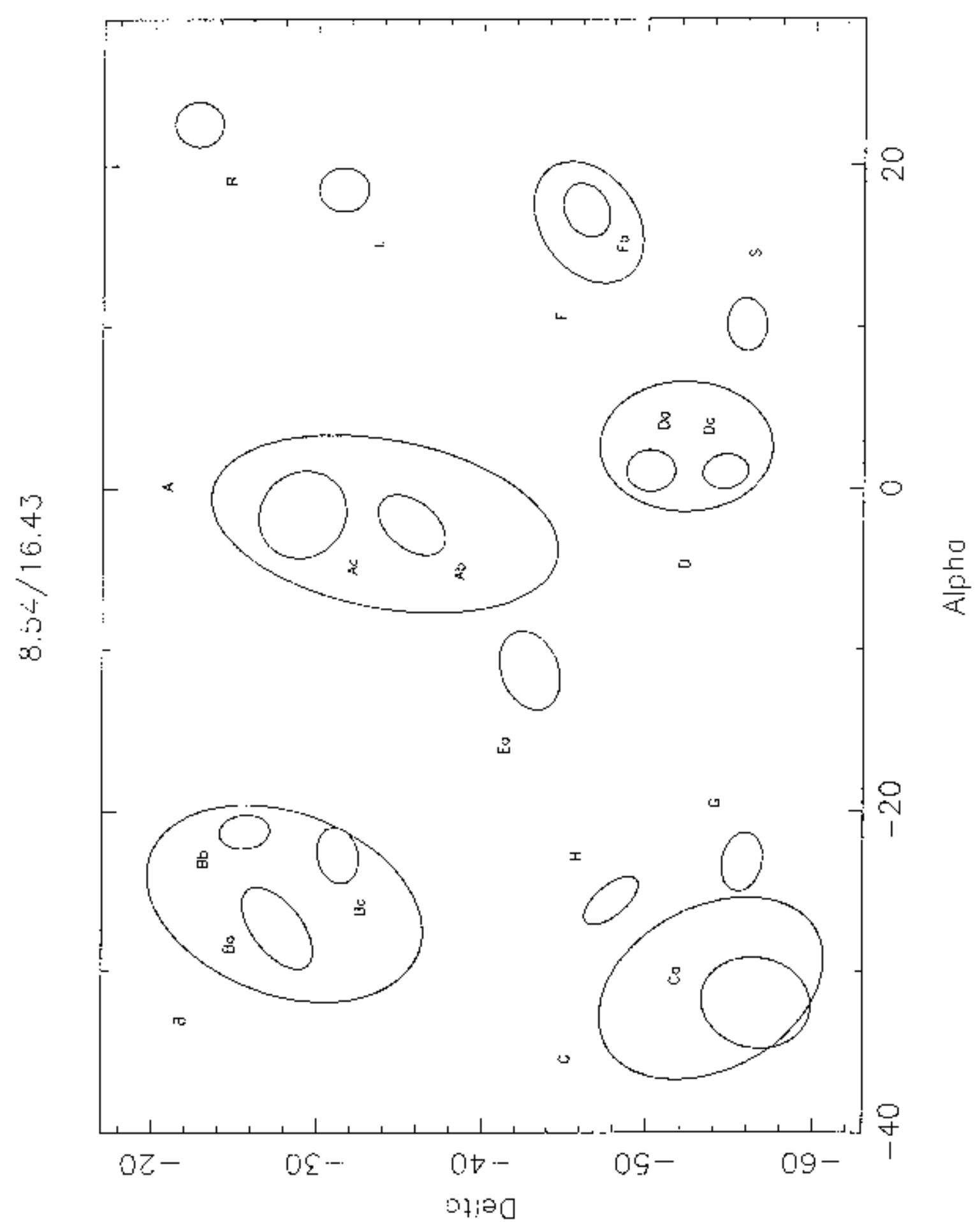

Fig. 1. b) Representation of the structures detected in the domain D085 


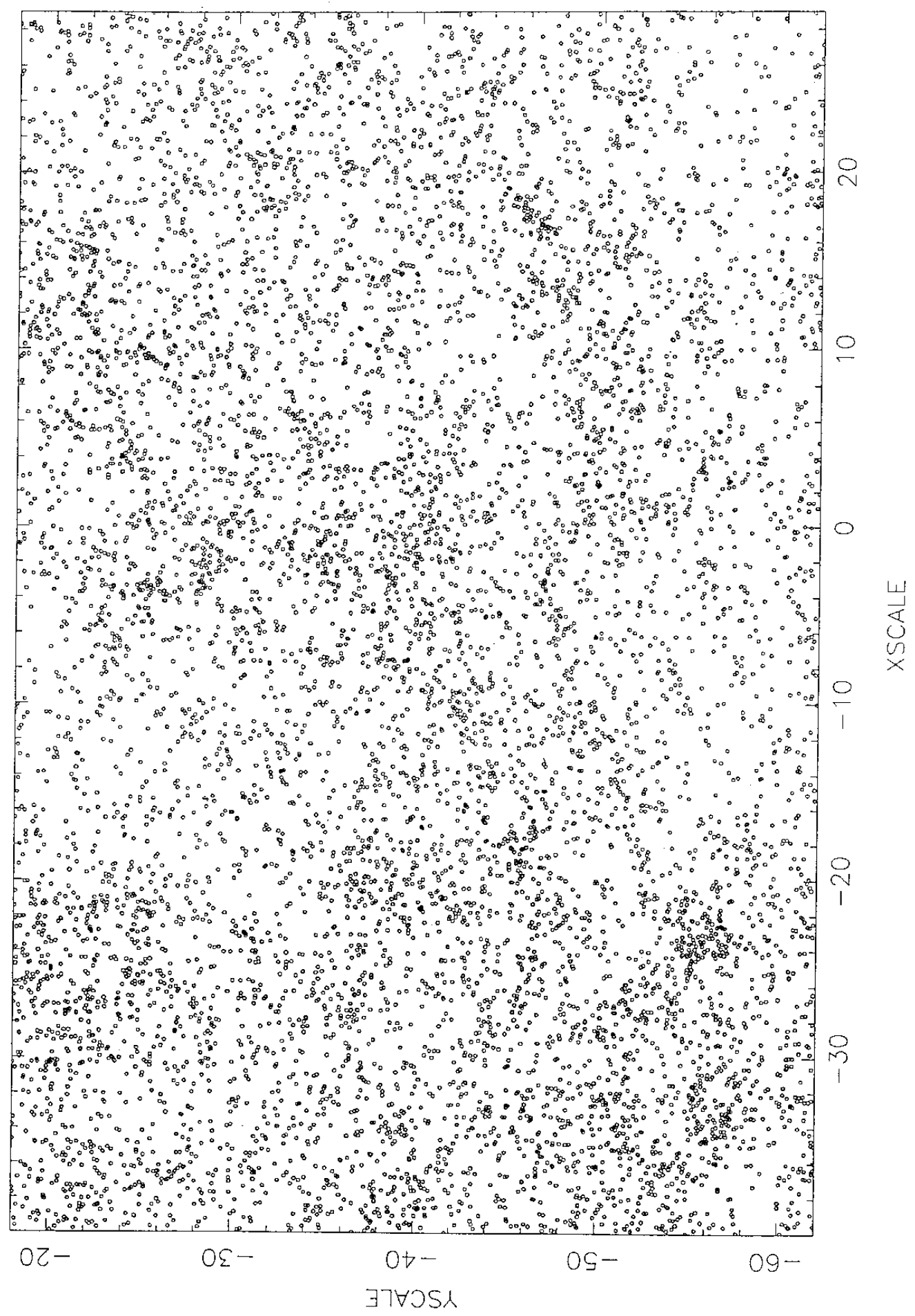

Fig. 2. a) Spatial distribution of galaxies in the domain D164 


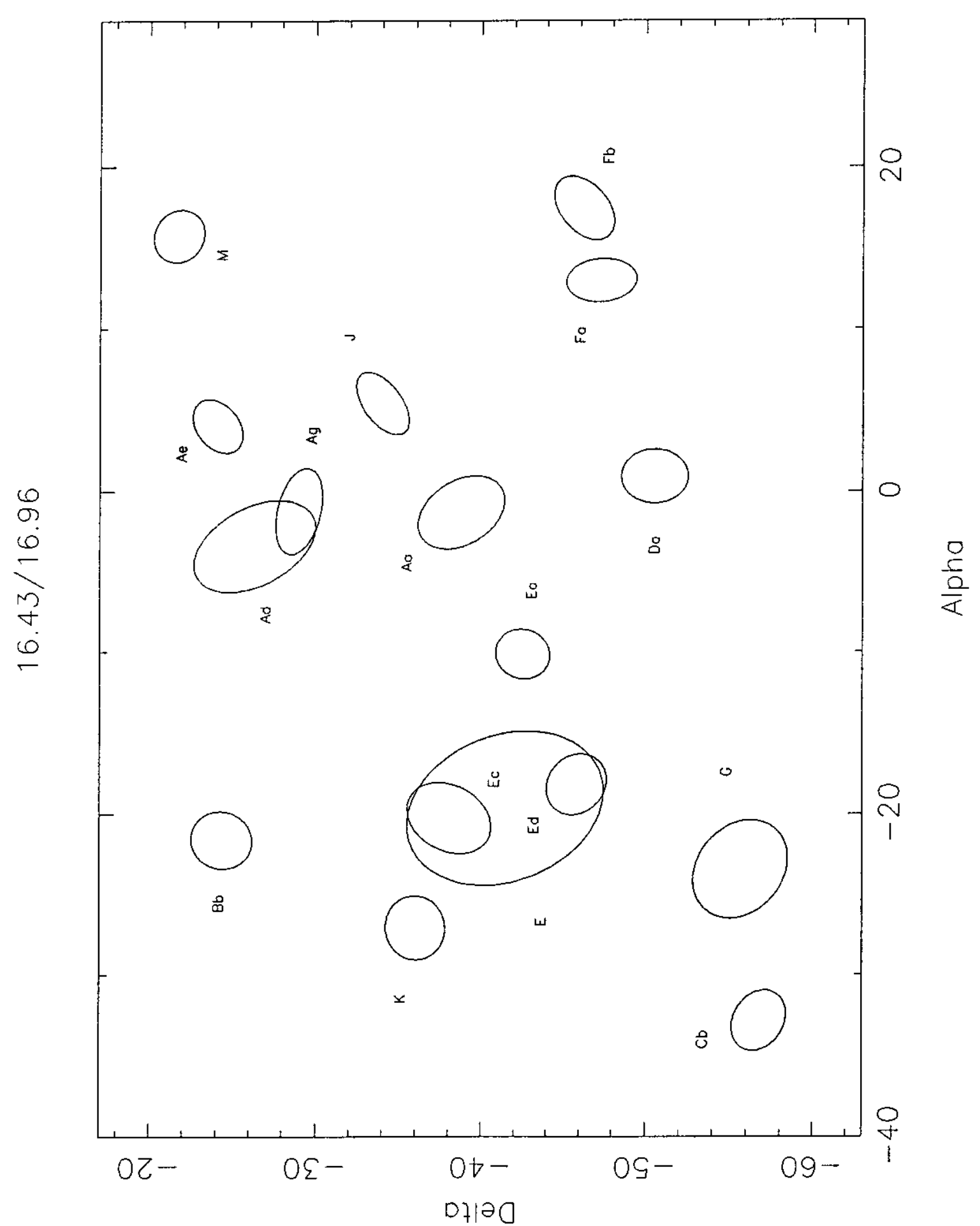

Fig. 2. b) Representation of the structures detected in the domain D164 


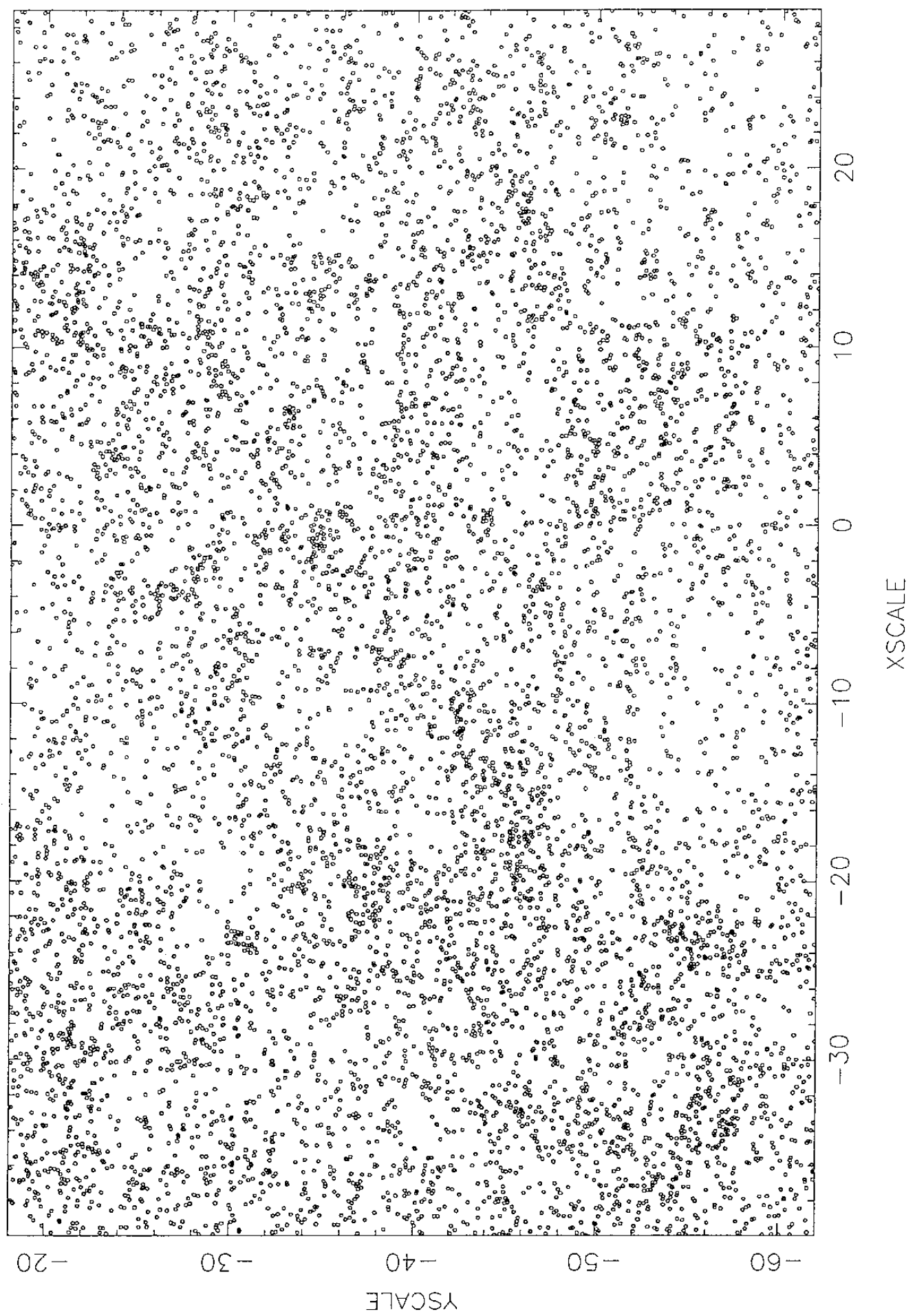

Fig. 3. a) Spatial distribution of galaxies in the domain D169 


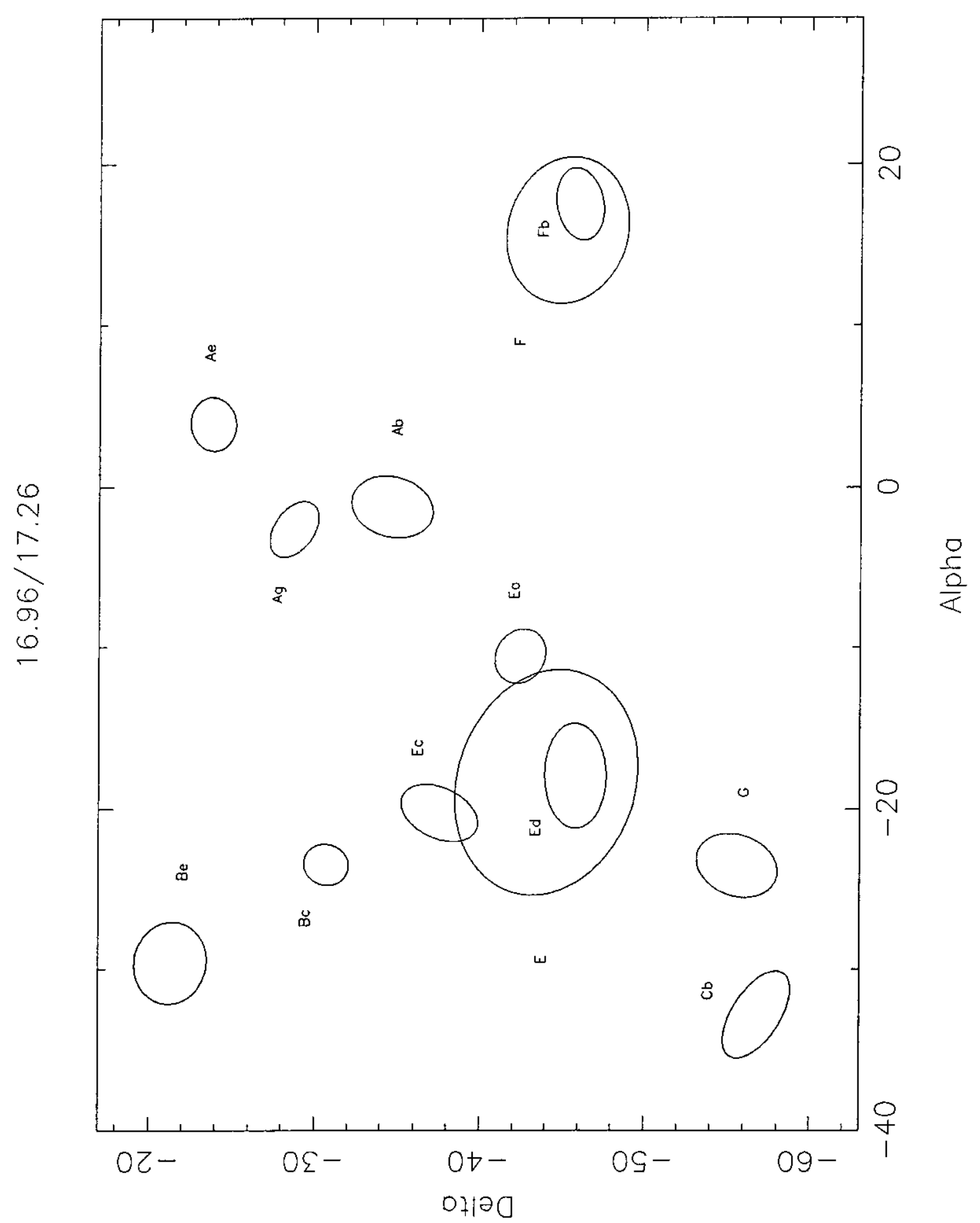

Fig. 3. b) Representation of the structures detected in the domain D169 


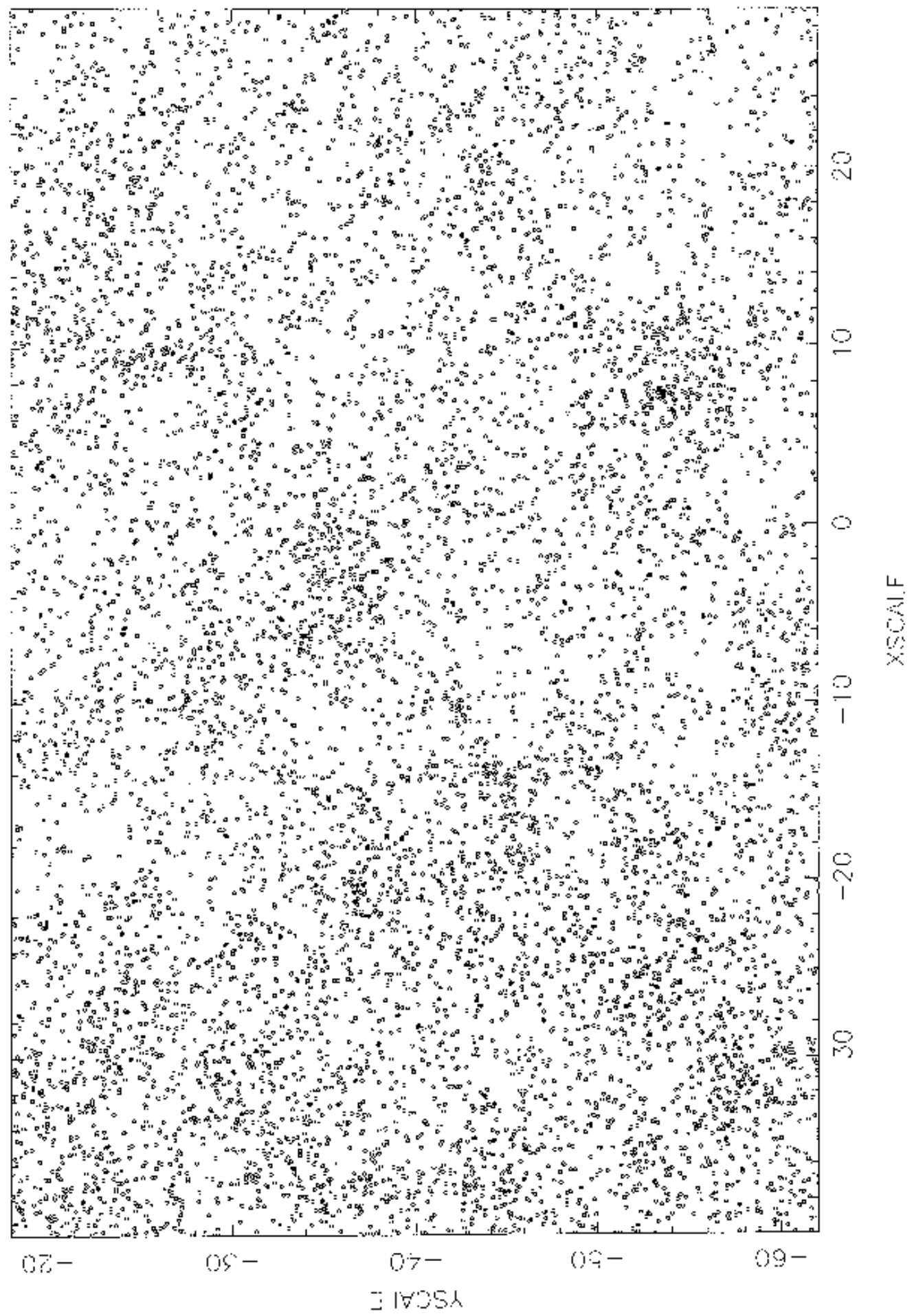

Fig. 4. a) Spatial distribution of galaxies in the domain D180 


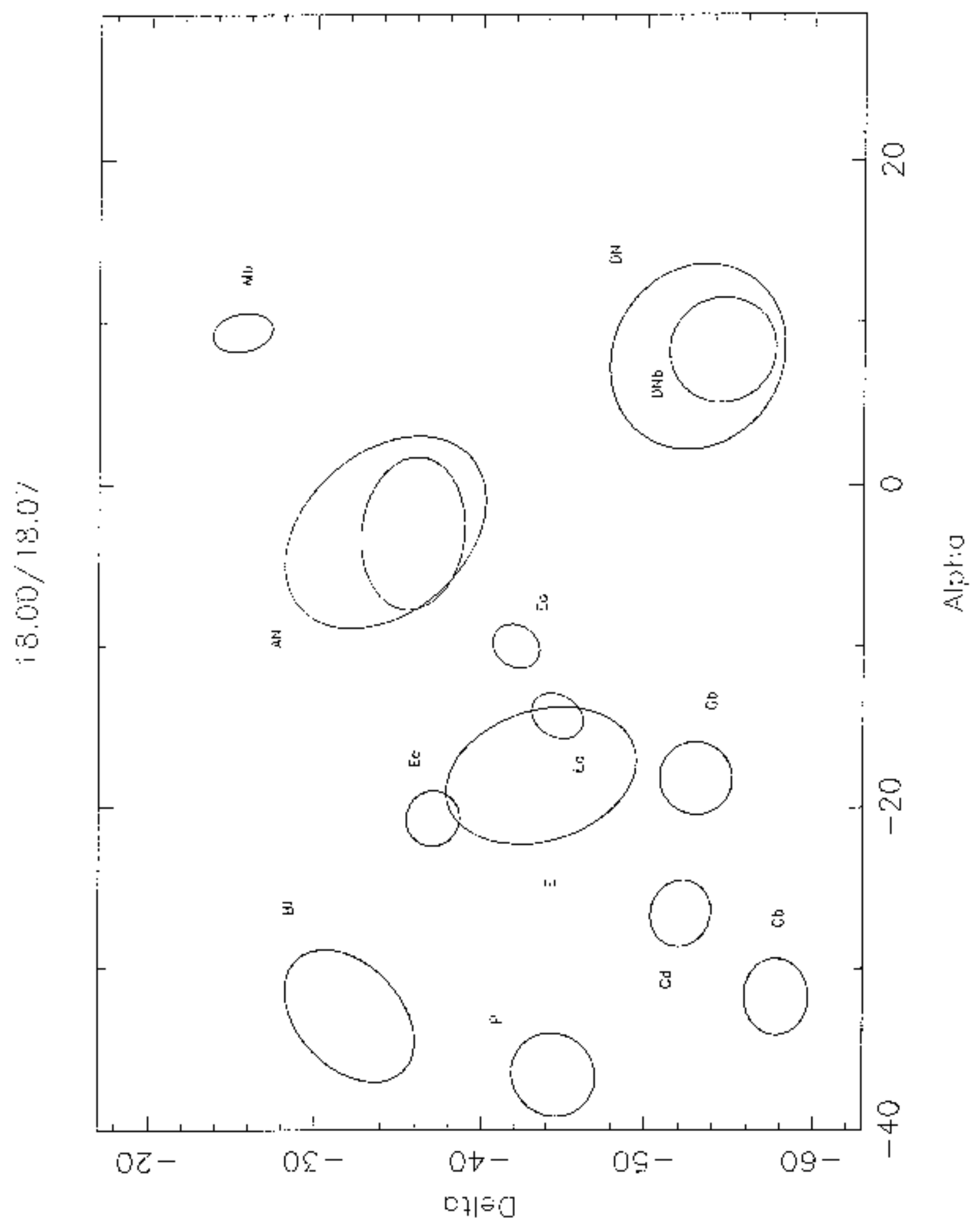

Fig. 4. b) Representation of the structures detected in the domain D180 


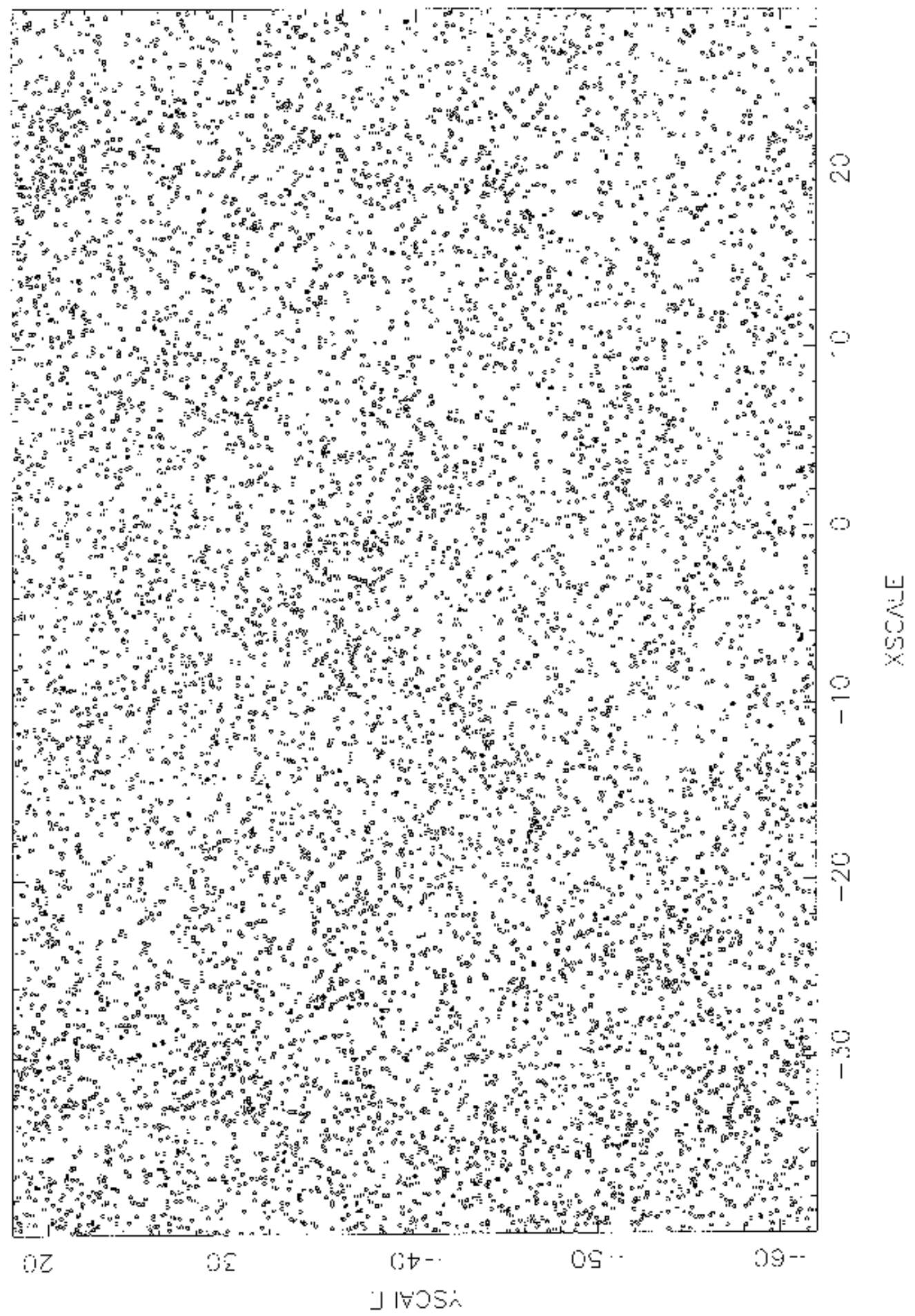

Fig. 5. a) Spatial distribution of galaxies in the domain D185 


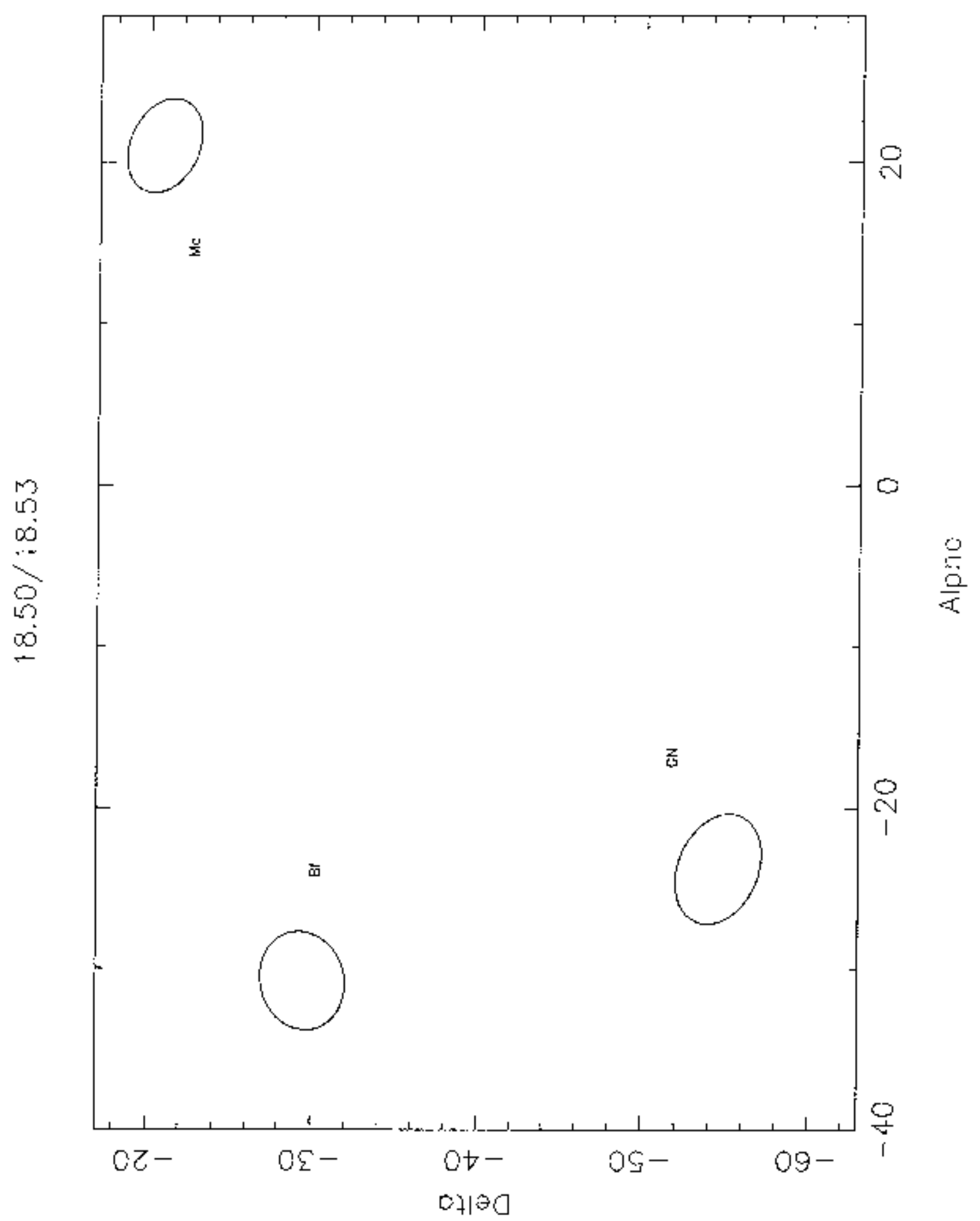

Fig. 5. b) Representation of the structures detected in the domain D185 


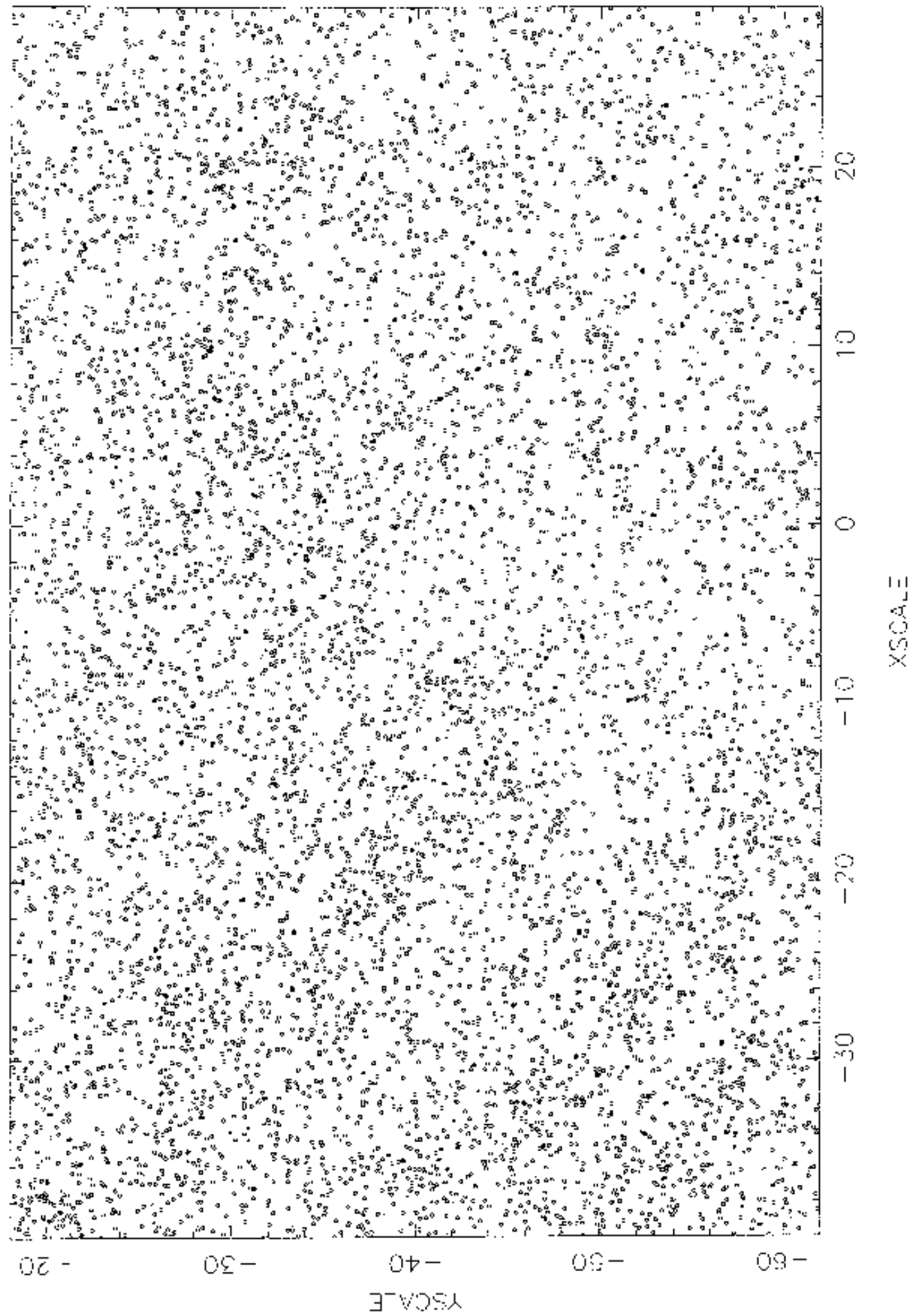

Fig. 6. a) Spatial distribution of galaxies in the domain D190 


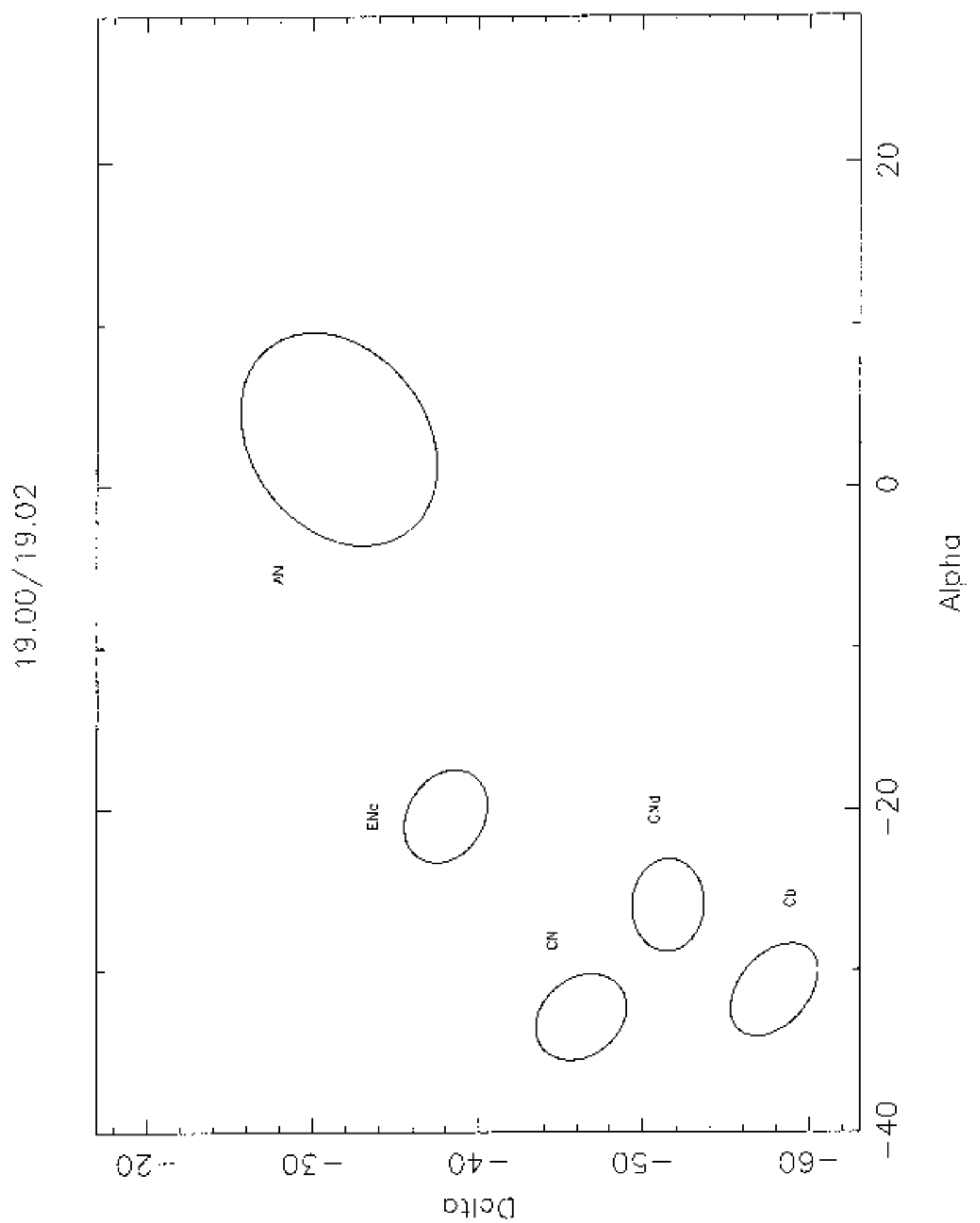

Fig. 6. b) Representation of the structures detected in the domain D190 


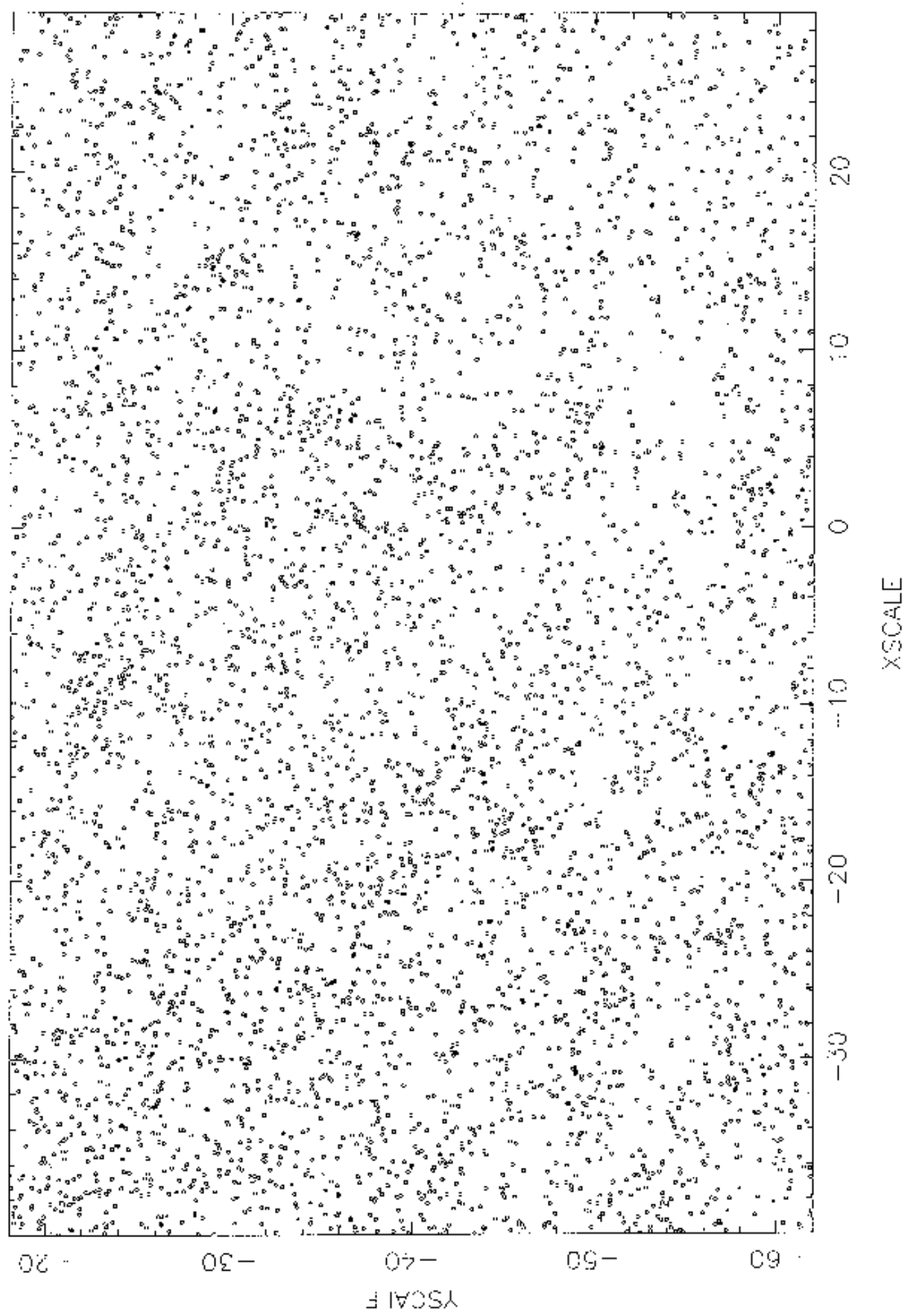

Fig. 7. a) Spatial distribution of galaxies in the domain D193 


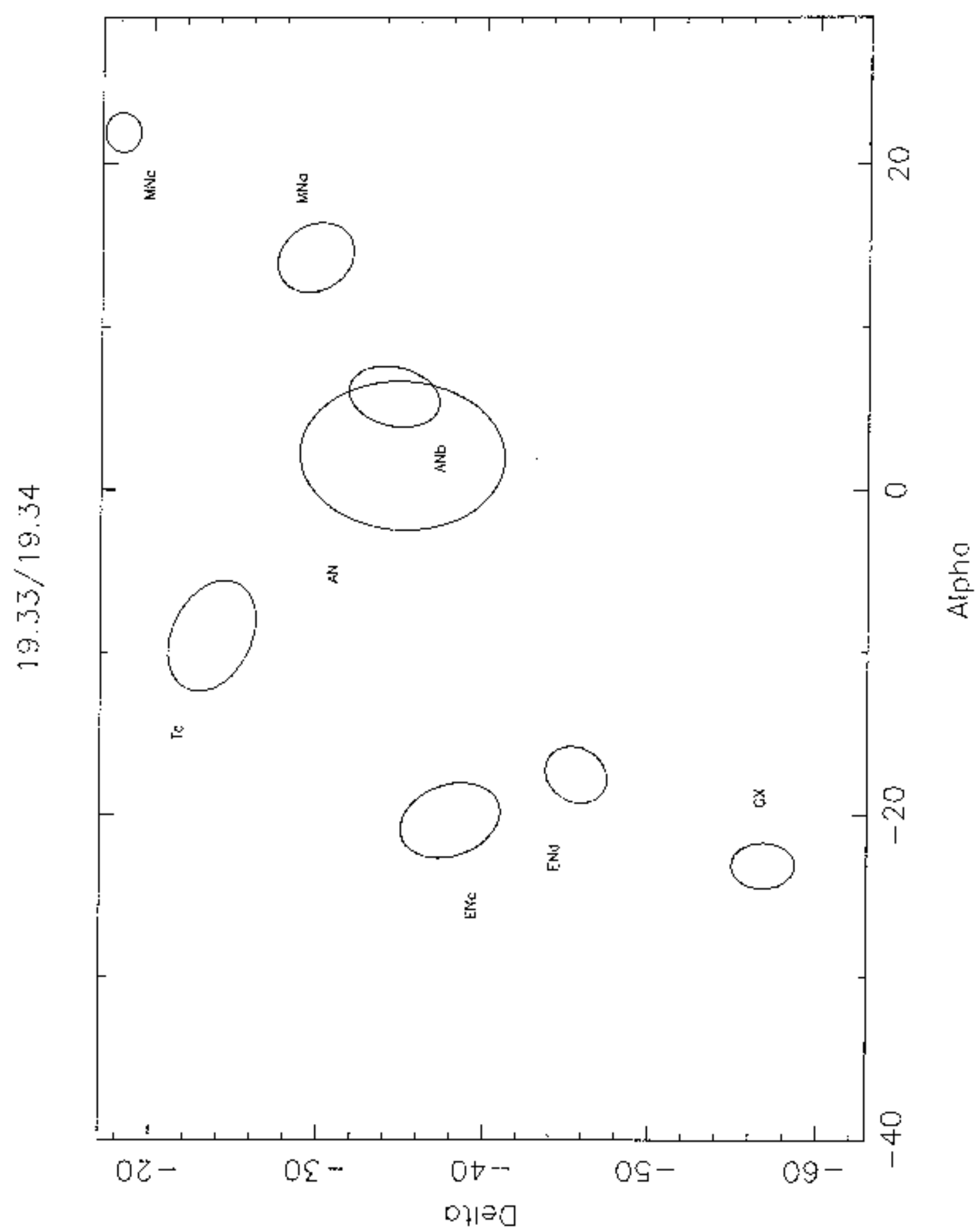

Fig. 7. b) Representation of the structures detected in the domain D193 


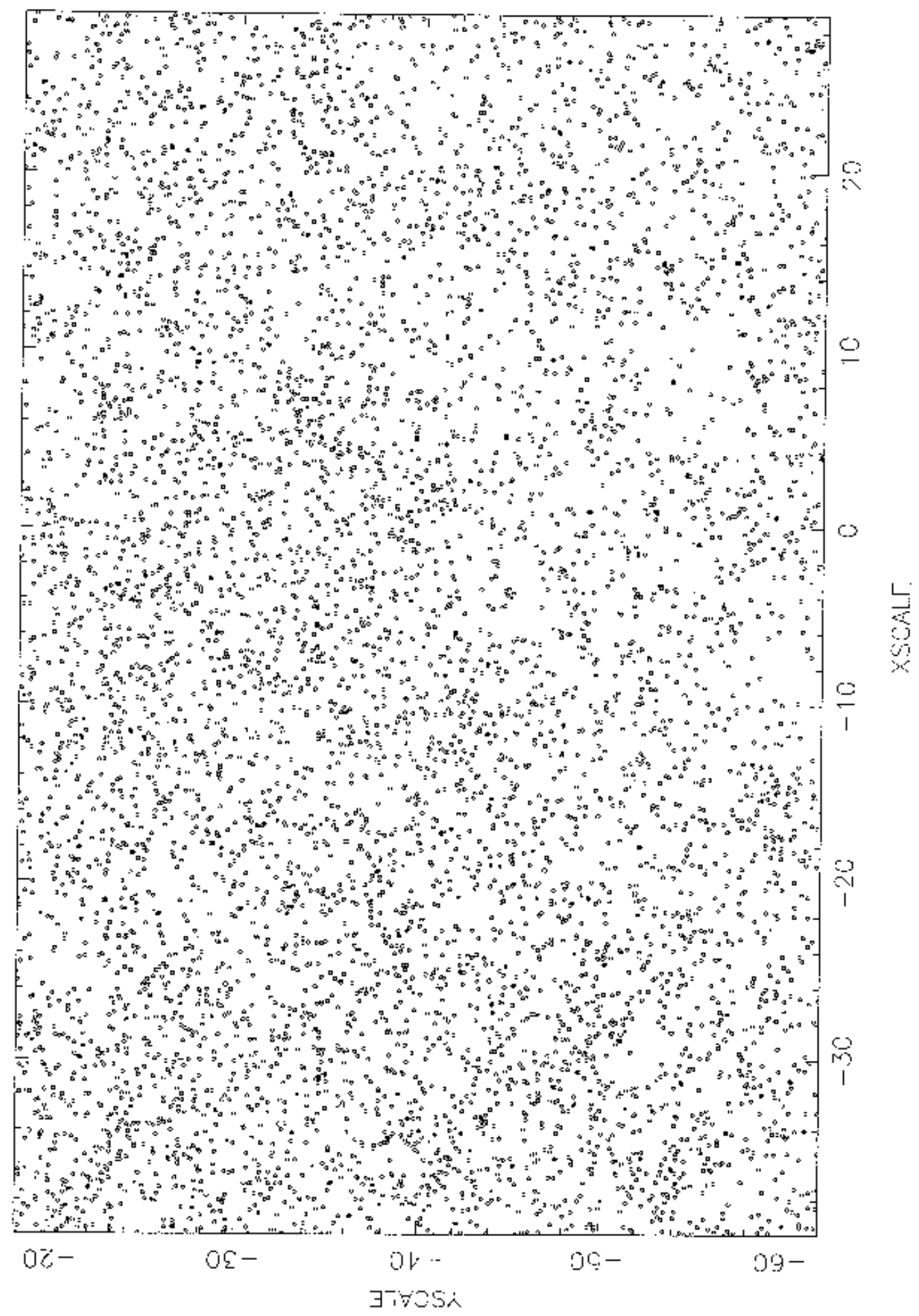

Fig. 8. a) Spatial distribution of galaxies in the domain D196 


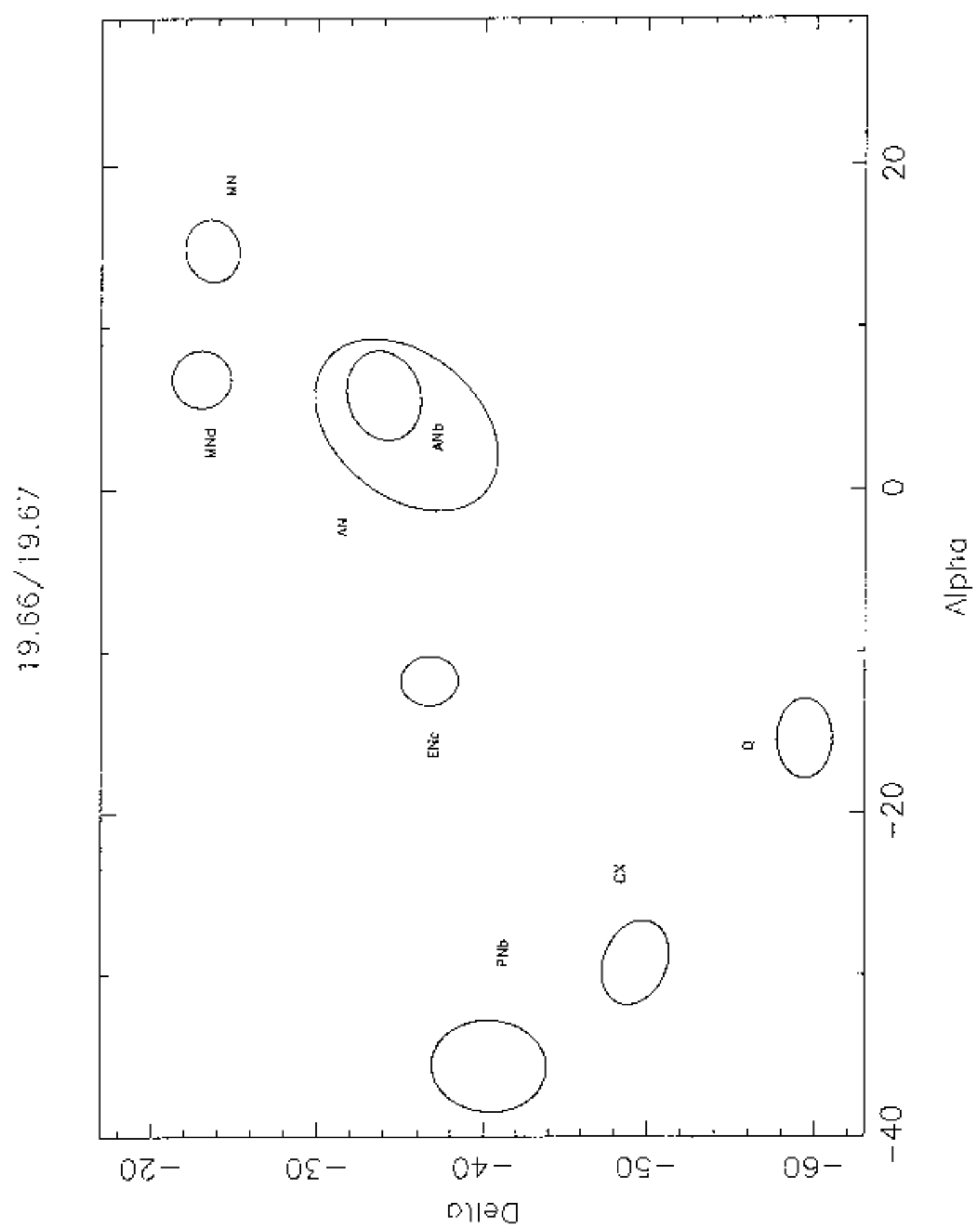

Fig. 8. b) Representation of the structures detected in the domain D196 


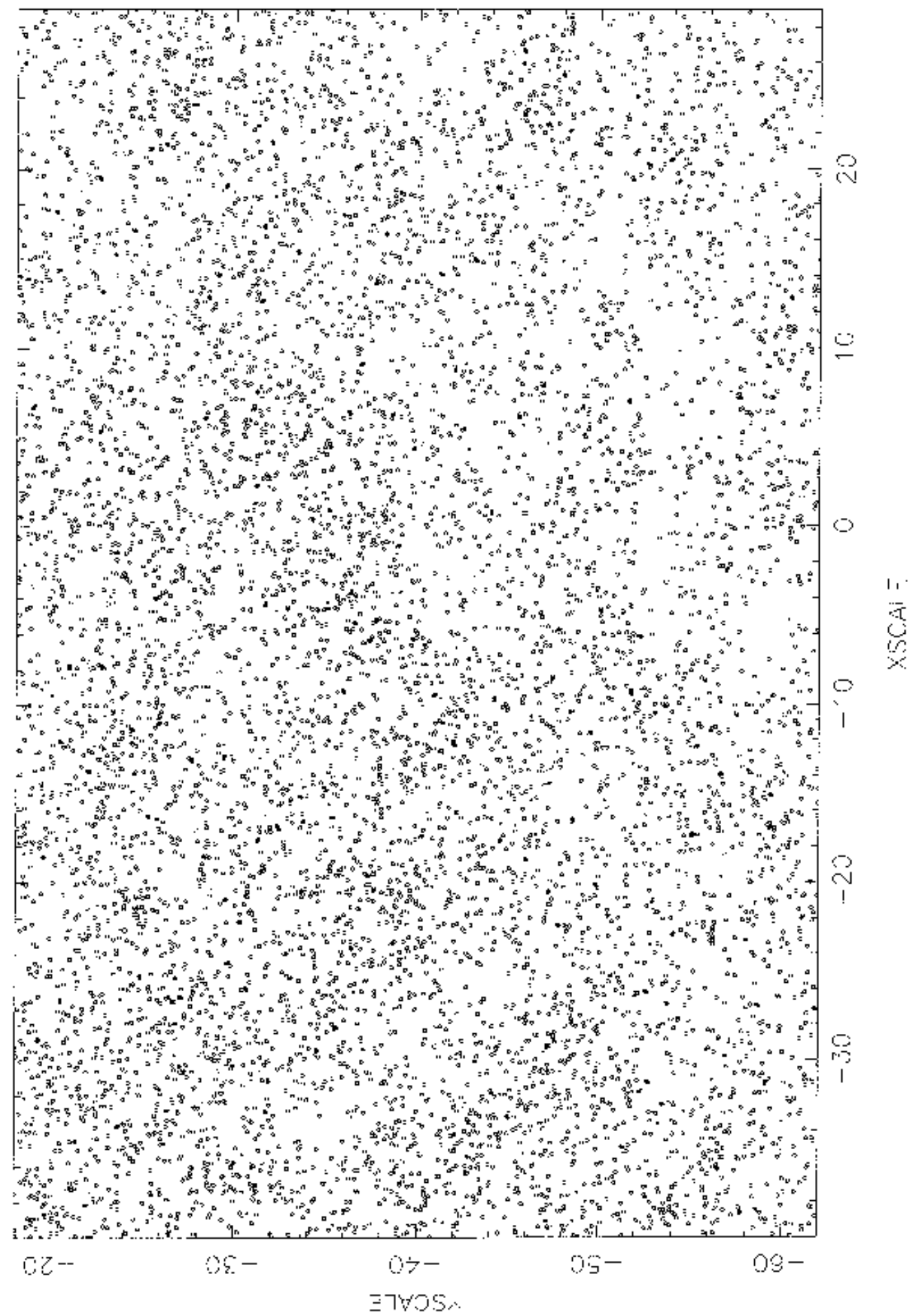

Fig. 9. a) Spatial distribution of galaxies in the domain D200 


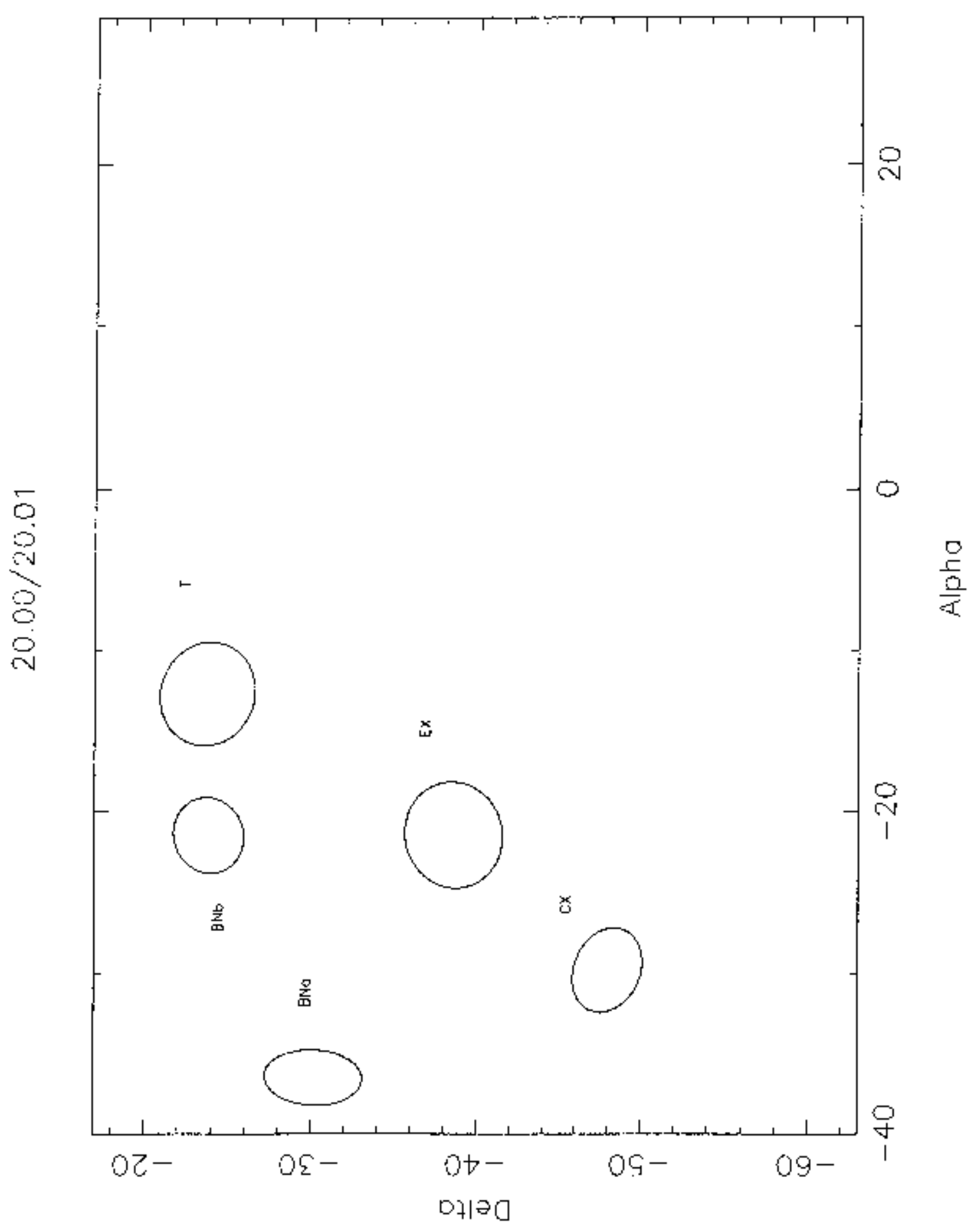

Fig. 9. b) Representation of the structures detected in the domain D200 
Fig. 10. a) Spatial distribution of galaxies in the domain D201 


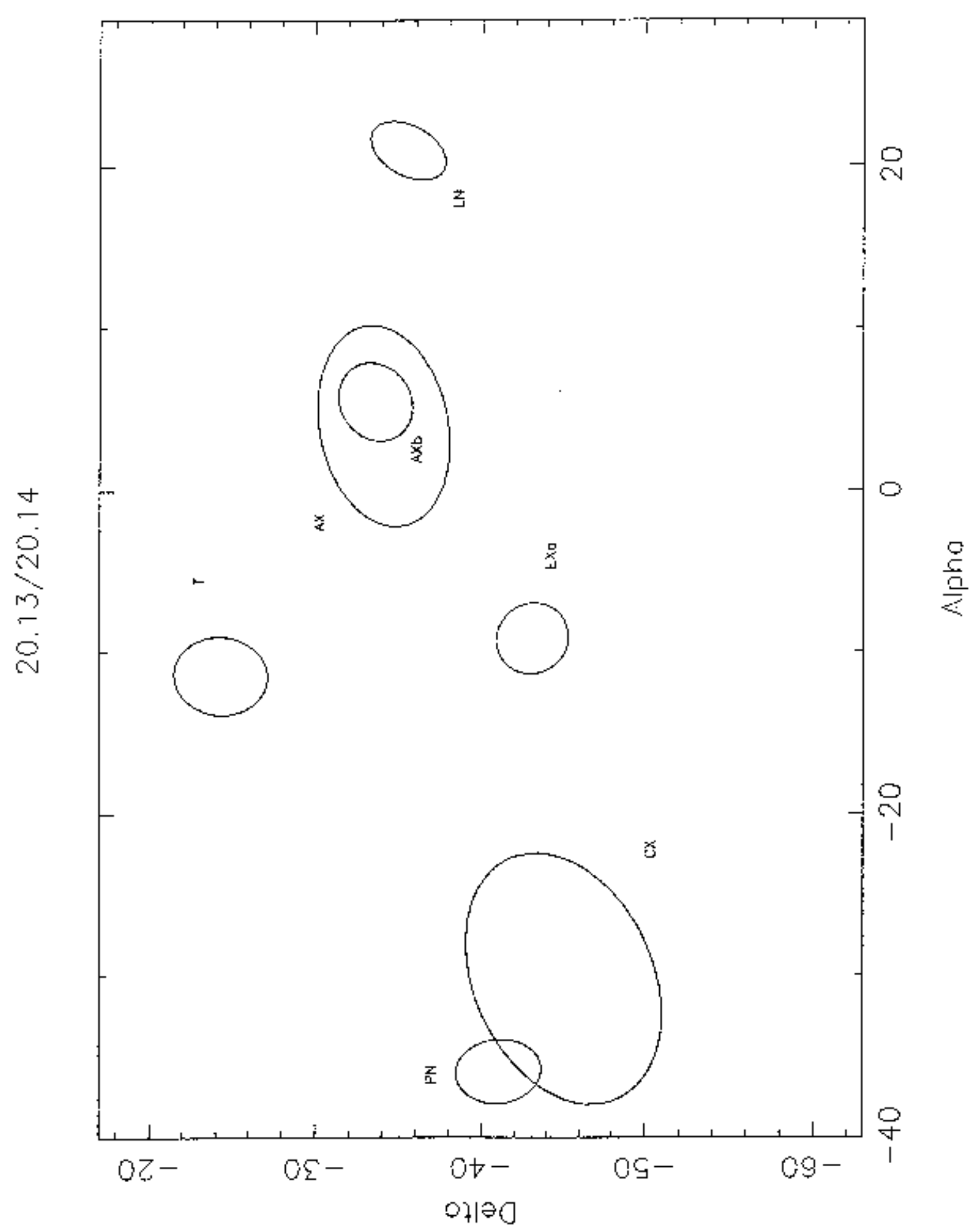

Fig. 10. b) Representation of the structures detected in the domain D201 


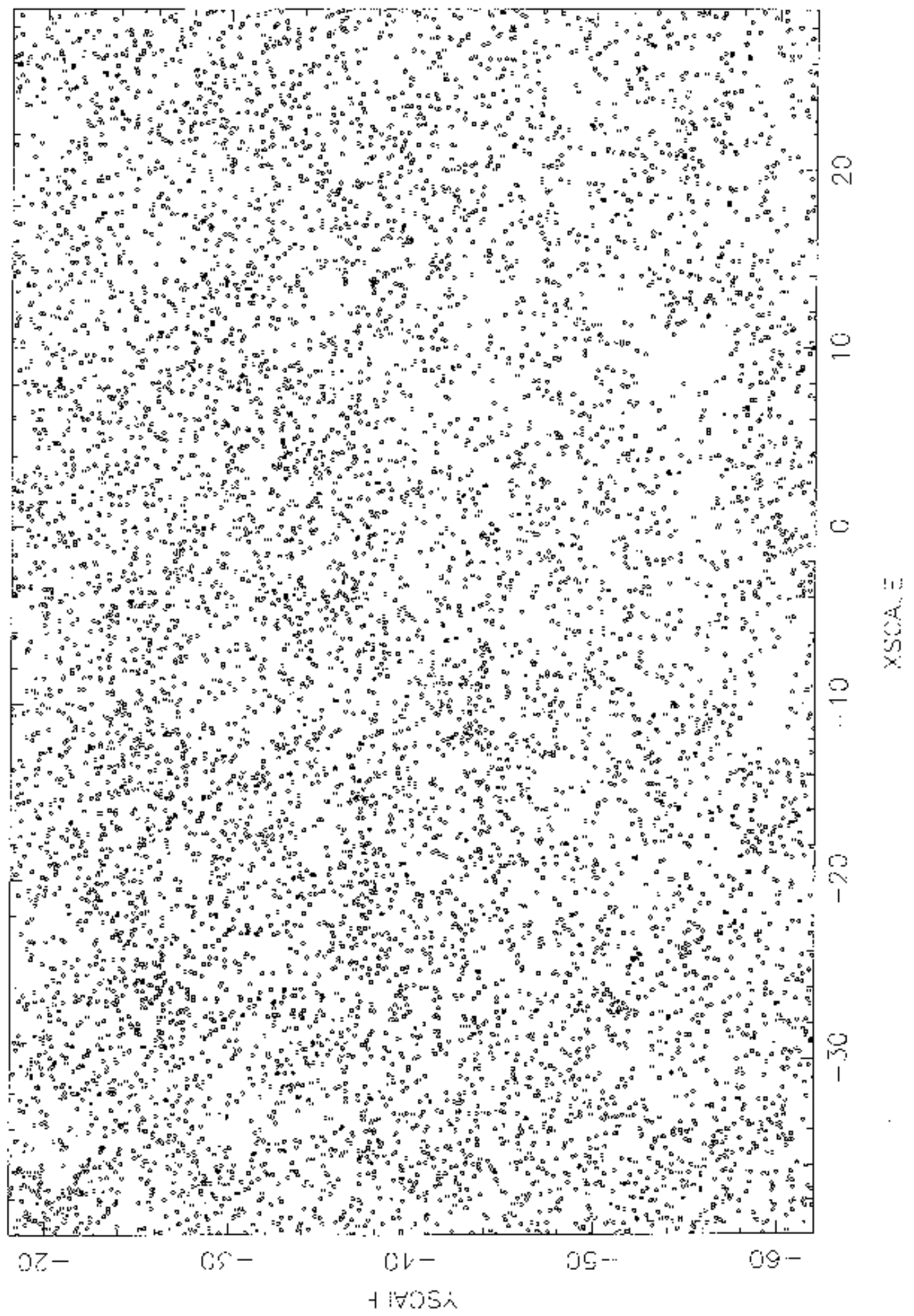

Fig. 11. a) Spatial distribution of galaxies in the domain D203 


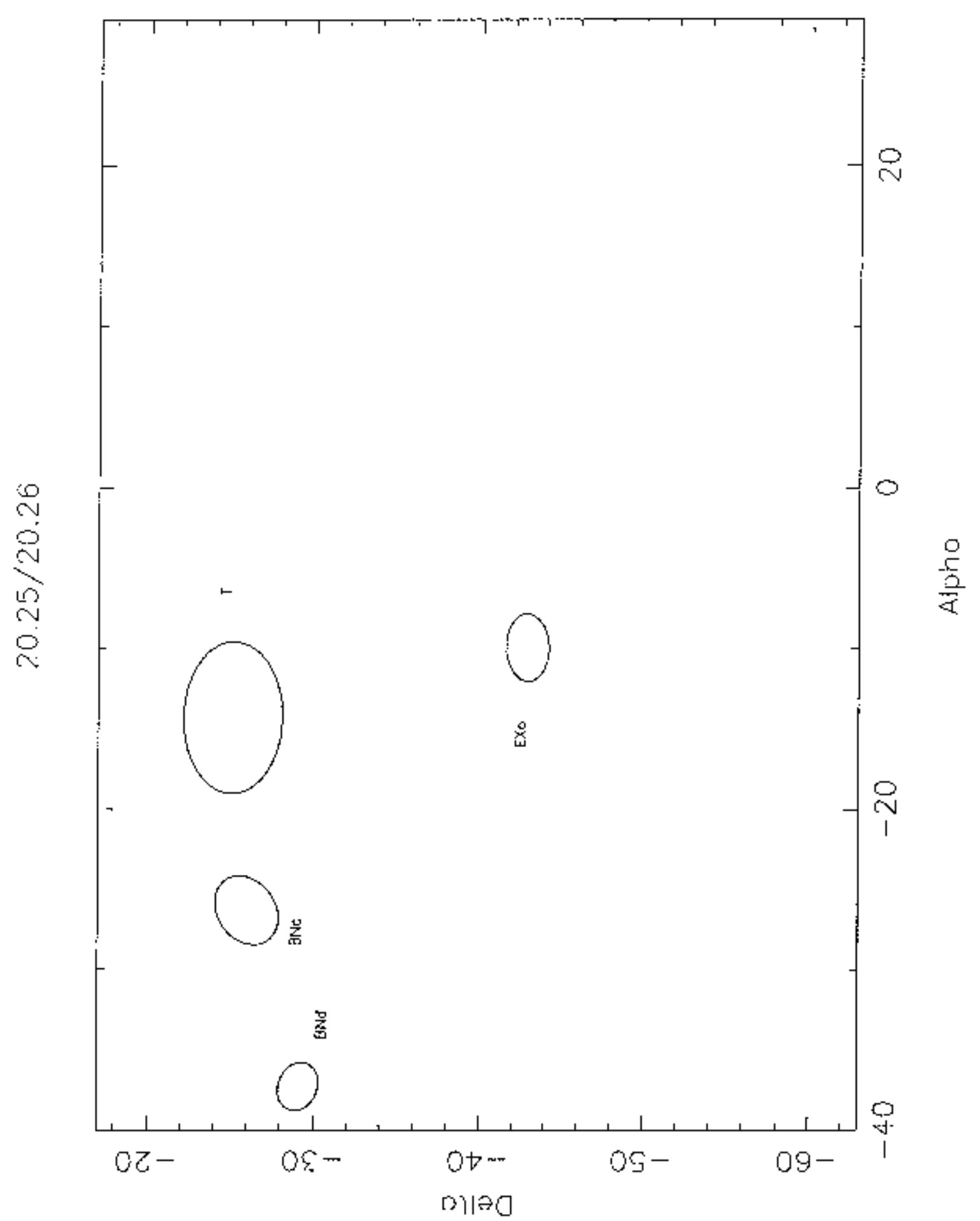

Fig. 11. b) Representation of the structures detected in the domain D203 


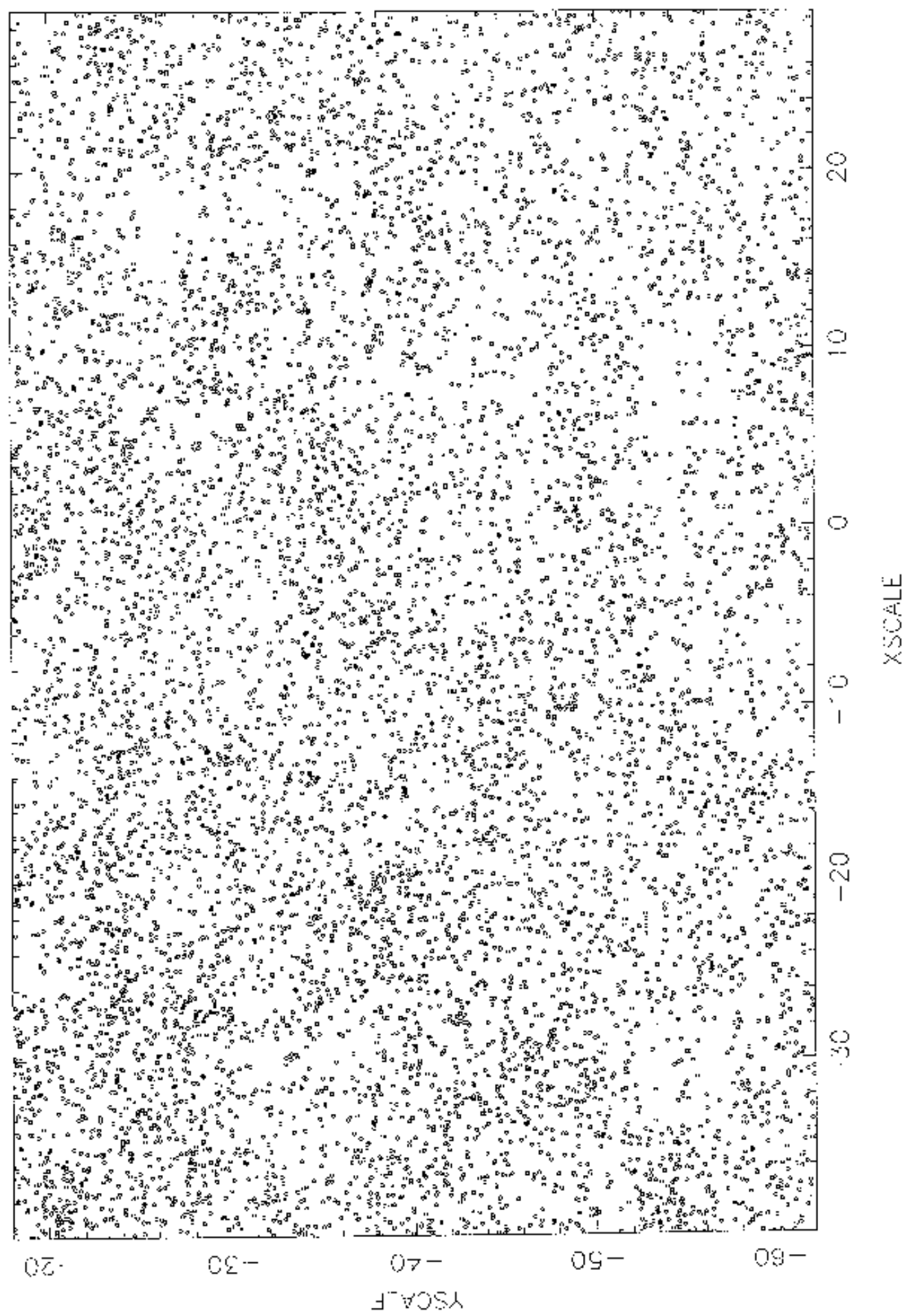

Fig. 12. a) Spatial distribution of galaxies in the domain D205 


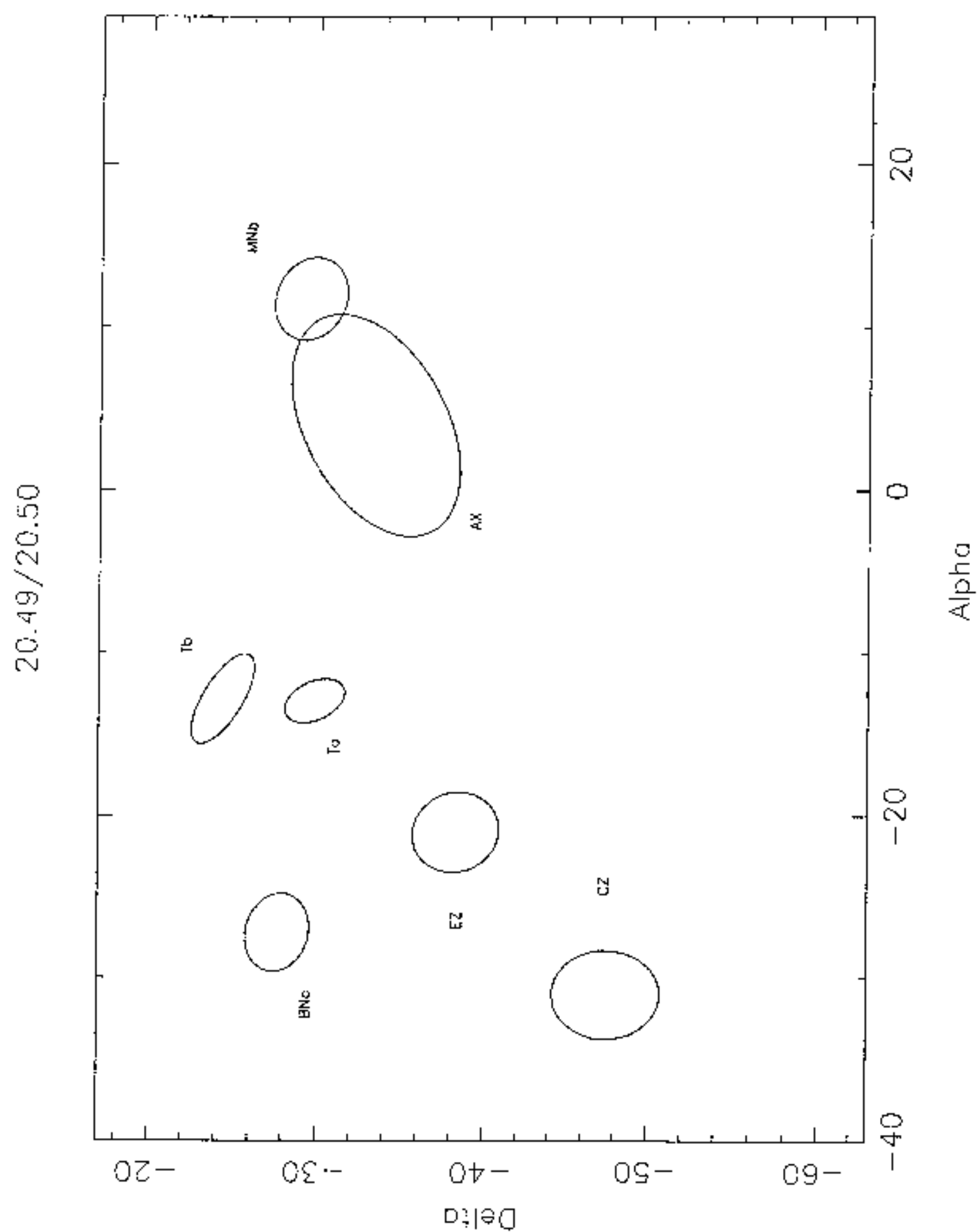

Fig. 12. b) Representation of the structures detected in the domain D205 
The main characteristics are listed in a series of tables. The direct output of the wavelet transform are given firstly in Table 2: position of the structures (right ascension and declination) and significance levels $P_{\mathrm{SL}}$. The error bars for all the positions given throughout this section are boxes of $10^{\prime}$. Next a parametrisation of the structures is included, giving the characteristics of the ellipses that fit their theoretical limits: position of the centroids, semi-axes, and orientations. As already stated, the position of the centroid may differ slightly from the position defined previously. The more the structure is irregular, the more the two definitions depart from each other, since the peak of maximum density is not geometrically located within the ellipse. Following on, in Tables 4 we introduce a morphological classification of the structures detected, following their relative extension and their relationship. Structures are listed for successive domains separately in order to allow a direct examination of the respective figures. The successive categories are those introduced in EMG:

- $\{\mathrm{W}\}=$ Oversized or "Ultra-large" structures

- $\{\mathrm{V}\}=$ Very large structures

- $\{\mathrm{S}\}=$ Superclusters.

- $\{\mathrm{E}\}=$ Intermediate or Elongated structures.

- $\{\mathrm{C}\}=$ Cluster-sized structures.

- $\{\mathrm{A}\}=$ Compact clusters or Associations.

The apportioning to a given category is discussed hereafter, in the light of the successive results. Obviously the distinction should come mainly from the true dimensions of the structures, for such information is not included in the present analysis. Nevertheless, we intend an approximate estimate of dimensions derived from the results obtained (see Sect. 4.4).

\subsection{Foreground domains: from 8.5 to 17.3 magnitude}

As commented above, the first three domains ([D085], [D164] and [D169]) are consecutive and so represent the complete set of available data for the region analysed. The choice of the two cut magnitudes (respectively 16.43 and 16.96) was arbitrary, and we checked that no detection is missed simply by producing intermediate images.

The balance of the structures found in this particular set is illustrated in Figs. 1-3 with results listed in Table 2. Our findings can be summarised as following:

- 1 Ultra-large structure ( $\{\mathrm{W}\}$-class $)$ of more than 10 degrees in extent;

- 2 Very Large structures $(\{\mathrm{V}\}$-class $)$ of 7 to 10 degrees in extent;

- 3 Large structures ( $\{\mathrm{S}\}$-class) of the order of 5 degrees in extent;

- 22 Intermediate structures ( $\{\mathrm{E}\}$-class), all but one related to the above superstructures;

- 9 Cluster-sized structures ( $\{\mathrm{C}\}$-class) mainly isolated in the field;

It must be noted that the topology evolves dramatically when considering different ranges of magnitudes. All the superclusters are exclusive to the brightest domain [D085], except E which instead is specific to the fainter ones. We can remark also that most of the minor structures involve galaxies of a limited range of magnitude since they appear in one domain only; others instead are equally present throughout successive domains so they involve a large range of brightnesses. This is the situation for Ea, $\mathrm{Fb}$, and $\mathrm{G}$ only.

When considering now the lower scales, we find many different outcomes. In particular, all the superclusters appear to have an irregular profile, since they systematically reveal minor structures inside; some of these substructures are multiple (e.g. in A, B, ) others instead are single core structures only (e.g. in C, F). Many minor structures finally are detected isolated, some close to a superstructure (e.g. G, J) but most completely independent as M, L, R, or $\mathrm{S}$.

All the above comments are seen recapitulated in Table $4 \mathrm{a}$, with the use of basic symbols to represent the connected events. The relationship stated between the structures is assumed in a systematic way: "included" and "involved" concern structures respectively fully, or partially, overlapping a minor one, and "related" concerns structures close to each other (less than 1 semi-major axis) but with no direct connection. Other cases are referred to as "isolated".

For each of the structures detected in these foreground domains we give also an estimate of the population i.e the number of galaxies involved. The population assumed to constitute a given structure is the excess of galaxies (E1) relative to the local background. In practice, we compute the total number of galaxies contained in the area defining the structure and remove the corresponding background estimated by exploring an area of 5 times greater. We also include a quantity $\delta n$ that should estimate such populations in terms of Poissonian fluctuations. If $N_{r}$ is the local background, then $\delta n$ is simply E1 given in units of $N_{r}^{1 / 2}$. Since E1 is an excess, values greater than 1 still identify high relative densities. For a given structure, the value of $\delta n$ can also be regarded as a non-integer "richness".

The results are listed in Table 3 . We can remark that all the structures are assigned relevant populations consistent with the significance levels $\left(P_{\mathrm{SL}}\right)$ given through our procedure. It is noticeable also that the superstructures (A, B, and $\mathrm{C}$ ) are assigned very high populations relative to their own background. This can be considered as a bias, since (by definition) superclusters are surrounded by relative underdense regions, and which does not affect the high significance level for these large scale structures. 


\subsection{Intermediate domains: from 18.0 to 19.6 magnitude}

The next five domains are magnitude slices (subsamples [D180] to [D196]). As already stated, the position and depth of the slices in the magnitude axis are designed in order to retrieve regularly spaced intervals in terms of distance, as well as equivalent depths in true dimensions. No assumptions have been made, however, concerning any distance-magnitude relation. Our computation is extracted from observable parameters only. Within the limits of the dataset we can remark that the population $N$ increases with magnitude $m$ following a simple exponential law:

$$
N(m)=\mathrm{e}^{\alpha\left(m-m_{0}\right)}
$$

where $m_{0}$ is a given reference point on the magnitude axis. The main dataset is well fitted with a value of $\alpha=0.5$.

Let us now introduce $\lambda$, the evolution of the total population of the slice $\Delta N$ at the position $m$ :

$$
\lambda(m)=\Delta N(m) / \delta m
$$

$\lambda$ is a density per unit of magnitude, and it can be written also as:

$$
\lambda(m)=\omega_{m} \times \rho(m)
$$

where $\omega_{m}$ is a true density, i.e. population per unit of volume $\delta r^{3}$, and $\rho(m)$ is a relation between dimension and magnitude. Thus we have:

$$
\omega_{m}=C \times \Delta N(m) / \delta r_{m}^{3}
$$

where $C$ is a numerical constant, and

$$
\rho(m)=\delta r_{m}^{3} / \delta m
$$

$\lambda(m)$ is directly measured from the dataset by considering consecutive magnitude slices of a constant depth (e.g. in this work $\delta m=0.05$ ). Then we assume that the true density $\omega_{m}$ does not change drastically with distance - for the present purpose the population of a given largeunit of space is the same everywhere, and hence it should not depend on the magnitude. We consider it to be a constant and denote it $\omega$. From the above relations it follows that we can derive the value of $\rho$ :

$$
\rho(m)=\lambda(m) / \omega
$$

Thus, the quantity $\lambda(m)$ gives an acceptable estimate of the local relationship between dimension and magnitude $\delta r_{m}$ :

$$
\delta r_{m}=K \times \lambda(m)^{1 / 3}
$$

(where $K$ is a constant which includes $\omega$ ).

The aim of the above computations is not to determine the real distances $r_{m}$ - that is the purpose of redshift measurements. Here we only obtain an estimate of the relative dimensions $f$ from one domain to another, in order to keep the correct hierarchy of the sizes in apparent dimensions (degrees) of the structures detected: $f_{i}=$ $\left(\delta r_{m}\right)_{i} /\left(\delta r_{m}\right)_{i-1}$.

Hence we take a given magnitude slice as reference and compute the relative distance $D_{i}$ of the following slices $i$, in the form of $D_{i}=f_{i} \times D_{\text {ref }}$ where $D_{\text {ref }}$ is the distance of the chosen reference domain; $f_{i}$ is also the factor applicable to the dimensions when comparing structures through different domains. In that way $\theta$ degrees in a given domain $i$ is more likely equivalent to a dimension of about $f_{i} \times \theta$ degrees in the domain of reference. The advantage of such an estimator is that it comes from observables only:

$$
f_{i}=\left(\Delta N_{i}(m) / \Delta N_{i-1}(m)\right)^{1 / 3}
$$

Table 1 lists the resulting values of $f_{i}$, with the first magnitude slice [D180] taken as reference.

Figs. 4-8 illustrate the structures found in this set, with the corresponding results listed in Table 2. An account of the detection is summarised as follows:

- 1 Ultra-large structure ( $\{\mathrm{W}\}$-class $)$ of more than 6 degrees in extent;

- 5 Very Large structures ( $\{\mathrm{V}\}$-class) of up to 6 degrees in extent;

- 16 Large structures ( $\{\mathrm{S}\}$-class) of the order of 3 degrees in extent;

- 13 Intermediate structures ( $\{\mathrm{E}\}$-class) of up to 2 degrees in extent;

- no compact structures.

We can remark that all the structures detected in this set are new, except some in the E-region which represent the continuation of a group of structures found in the previous foreground domains. The first domain [D180] is rich and complex, and it appears totally disconnected from the following domains. The intermediate domain [D185] is poor in structures, appearing as a frontier with the successive regions ([D190] and beyond). These show again many new features, all being unrelated to any previous one.

Regarding the relationship between the different classes of size, we can see that structures appear mainly isolated in the three dimensions (i.e. position in its own field and within the successive domains). Only two largescale structures (AN and ENc) are present in three successive domains, and this implies that very large extents are covered. Contrary to what happens in the foreground samples, we do not assist here any detection of lower scales structures directly split from large scales ones. All these results are recapitulated in Table $4 \mathrm{~b}$, with the same symbols as used in the previous section.

\subsection{Background domains: from 20.0 to 20.5 magnitude}

These domains represent the last 4 magnitude slices (subsamples [D200] to [D205]). The major characteristic of 
such remote domains is the presence of excessively extended features, namely the Ultra-large structures. This however can be considered as a bias of the procedure for detection: the maximum scale explored is $\sim 0.25$ times the size of the available field, due to the necessity of considering a significant local background (e.g. for computing the confidence levels). In this work the limit corresponds to approximately 10 degrees. Obviously such an upper limit is much less restrictive with the background domains than with the foreground ones, due to the distance effect. Thus the detection of the Ultra-large structures is suitable in the background samples where the distance effect is high (see the rates $f_{i}$ in Table 1), with apparent sizes not much exceeding 10 degrees. But detecting equivalent features in the "bright" samples would require the consideration of a much more extended dataset. In the same way clustersized structures get lower apparent sizes in such remote domains, hence their detection appeals to small-scale investigations and is discussed, therefore, in the next section. Both the absence of extended structures in the foreground domains and the absence of cluster-sized structures in the present background domains are not to be considered as a physical effect.

Figures 9-12 illustrate the detection results, with characteristics listed in Table 2 . The balance of the structures found in this particular set is summarised as following:

- 4 Ultra-large structures ( $\{\mathrm{W}\}$-class) of more than 4 degrees in extent;

- 13 Very Large structures ( $\{\mathrm{V}\}$-class) of up to 3 degrees in extent;

- 2 Large structures ( $\{\mathrm{S}\}$-class) of the order of 2 degrees in extent;

- no intermediate or compact structures.

The most relevant event is the structure $T$ which extends throughout the whole range of magnitudes, splitting at the end into two smaller features. Otherwise the relations between structures is again very limited, with few items slightly superimposed on each other. No complex hierarchy is observed except the $T$-region and another supercluster (AX in [D201]) which contains also a core structure. This is recapitulated in Table 4c.

\subsection{Summary of the wide-field detection}

In the main field we find a total of 87 structures, arranged as follows:

- 6 Ultra-large structures ( $\{\mathrm{W}\}$-class);

- 19 Very Large structures (\{V\}-class);

- 19 Superclusters (\{S\}-class);

- 35 Intermediate structures ( $\{\mathrm{E}\}$-class);

- 8 Cluster-sized structures (\{C\}-class).
The full detection results for the 12 domains is drawn together in Table 5, where the essential characteristics mentioned in the previous sections are summarised, with the structures presented following the right ascension.

\section{Small-scales detection}

When considering the detection on small scales, we are allowed to take into account the whole available data since both the sizes of the corresponding analysed fields and the number of galaxies concerned remain reasonable quantities for the automatic procedure.

The principle of sampling in volumes is opposite to the previous procedure for magnitude samples: the advantage of the volumes is the completeness, so the structures are detected through their entire population. The inconvenience a priori is the projection effects, for at the scales concerned these remain at an acceptable level: the extension of the individual subsamples is limited enough so that the probability of a rigorous superposition is low. The transition with the previous wide field detection is assured by considering intermediate scales as well, i.e. the sizes of the lower structures detected in the magnitude slices ( $\sim 1$ degree). Hence some structures of those sizes may have been missed by the procedure of the magnitude slices (e.g. lying partially out of the magnitude range), but they will be fully detected in the present volumes. The partition in volumes has been constructed from a preliminary small scales analysis of thin magnitude slices. Such a rapid detection highlights the regions containing an event (structure) and a volume is subsequently defined encircling. This procedure avoids most of the projection effects, since structures of distinct classes of magnitude are kept in different volumes so they do not interfere with each other. Sometimes several structures are grouped into a unique volume in order to limit the amount of subsamples to be analysed; This is done whenever possible, i.e. when structures are close but separated enough that they do not introduce confusion. And finally, the confidence levels are computed in the same way as for magnitude slices, i.e. by a comparison with Monte-Carlo simulations which are exact replicas (limits, populations, etc.) of the successive analysed samples. Our significance levels $P_{\mathrm{SL}}$ are calculated for all the structures detected, including those already found in the magnitude slices.

In the following we present the results organised into 10 "zones" labelled respectively "A" to " $\mathrm{T}$ ", each corresponding to a particular region of the field. Then labels are attached to the volumes according to their belonging to a zone (e.g. generic character VA for all the volumes of the "A" zone) and they also appear in the denomination of the detected structures. For, again, no relation should be systematically deduced between structures of a volume or zone: such a global partitioning has been made necessary due to the large number of structures, and the only purpose is to clarify the form of the results (tables, figures) 
not to presume any coherence in positions. The choice of generic characters (VA, VB, VC, VD, VE, VF, VL, VM, $\mathrm{VP}$, and VT) is, however, an approximate reminder of the superstructures revealed in the previous magnitude slices: e.g. the "A" zone (volumes VA) points toward the region of the $A$ superstructures. Then, an additional zone (labelled "V") is appended, but the successive VV volumes do not correspond to any zone: these latter merely group those loose structures not associated to any of the 10 major regions.

In Table 6 we give the list of all the volumes that contain relevant structures. Those devoid of any significant detection are not listed, which makes a total of 51 volumes. The denominations are formed by the above mentioned generic labels followed by a number. Three volumes, too extended for a direct analysis, have been split into two subsamples (labels 10 and 15 respectively) in terms of magnitude range.

\subsection{Detection results: Figures and tables}

In Figs. 13-23, we present the main results of the multiscale detection throughout the whole dataset, in the same form as previously: viz. for each structure detected an ellipse is fitted to the isophote of the wavelet coefficients that delimits its extent. The overlapping ellipses denote physically related structures, or instead structures found independently but in superposed volumes (see hereafter). The figures correspond to the 11 respective zones, with the addition of Fig. 24, which is a "main view" of the smallscale detection of the whole field: all the structures are represented together, for this figure is able to be directly superposed on the previous magnitude slices and therefore allows us to compare the respective distributions of small-scale and large-scale structures.

In the corresponding series of tables we give the output characteristics of the structures detected, presented in the same way as for the large-scale analysis (the significance of such characteristics are discussed in Sect. 4). All the tables describe the subsamples to which the structures belong, together with a numerical correspondence with the figures concerned. Thus, in the cases where ellipses overlap we can easily distinguish between true relationship and optical superposition, simply by checking if the structures concerned were found in the same volume or not. The complete list of the structures is given in Tables 7 , with the provided position of the centre (peak of the local wavelet coefficients) and the individual significance levels $P_{\mathrm{SL}}$. We still only retain those structures with $P_{\mathrm{SL}} \leq 0.050$, which means less than $5 \%$ chance of being due to random fluctuations. The error bars for all the positions given throughout this section are boxes of $1.5^{\prime}$.

This list introduces also the labels that are used in the following. We list also the parameters of the ellipses (centroids, axis, orientations). The individual populations of the structures are given in Tables 8: in particular the parameter $\delta n$ is assumed to estimate the real populations. Contrary to what happened with the large-scale analysis, populations are systematically computed here since the volumes are all complete samples. We only omit the cases of structures lying close to an edge where the local background cannot be estimated in a reliable way.

In the same spirit as for the magnitude slices, we attach to each volume a "distance factor". In this case, however, the samples are both too limited in extent and too wide in magnitude range to allow a computation of the previous parameter $f_{i}$. Hence, we only attribute to each volume an index $I_{\mathrm{M}}$, scaled from 1 to 6 , which states the hierarchy in the magnitude classes: from the main histogram of magnitudes of the whole dataset we simply decide on 6 zones equally populated, and each volume is associated with the zone that includes its own median magnitude. Obviously such an index cannot be used to derive the relative dimensions within the successive volumes, for it helps to keep under control the estimate of the true dimensions of the structures.

In Table 9 we present some comments on the structures, mainly the morphological categories attributed to each of them.

\subsection{Summary of small scales structures}

In the main field we find a total of 234 structures, arranged as follows:

- 11 Intermediate structures ( $\{\mathrm{E}\}$-class);

- 89 Cluster-sized structures (\{C\}-class);

- 134 Small-scale structures (\{A\}-class);

Only 10 of these structures were already detected in the previous magnitude slices $(4\{\mathrm{E}\}$-class and $6\{\mathrm{C}\}$ class). The full list is detailed in Table 10, with structures presented in order of right ascension increasing.

\section{Void detection}

When discussing "voids", one must be careful as to what exactly one is referring. In principle, voids are underdense regions within the spatial distribution of galaxies, i.e. regions of negative density profile relative to the local background. More usually, voids are defined as regions with a relevant lack of rich clusters or superclusters of galaxies, hence referring to the distribution of structures instead of that of the galaxies. This latter approach comes easily from a visual impression, and produces the extended voids claimed by many authors (see Einasto et al. 1994, for a review, also EMG and references therein).

The former approach instead is suitable for our method, since the wavelets treat the negative structures in the same way as the positive ones. The full procedure is applicable without any change, including the computation 
of the confidence levels; we just need to attend to the negative wavelet coefficients on the images. A prime result in such investigations is the total absence of small-scale voids; throughout the main dataset we only reported 3 intermediate voids, whose characteristics are listed in Table 11. As with the positive structures, positions and extents are those of the delimiting ellipses (all in degrees). Figure 25 provides a representation of these features. The two upper ones (OB, top, and OA, middle of the map) are detected in the 3 foreground domains, so they are present in the distribution of galaxies down to magnitude $B_{j}=$ 17.3. The lower void on the other hand (OC, bottom of the map) is exclusive to the first domain $\left(B_{j} \leq 16.4\right)$. Its own backgrounds are then fulfilled by the structures from the $\mathrm{C}$ and $\mathrm{E}$ zones. An important remark is that none of these 3 voids includes any of the small scales structures detected in the volume samples. When superimposing Fig. 24 ("all zones, small-scale detection") onto Fig. 25 (voids), some structures fall inside the void limits but these are largely background features involving galaxies at magnitudes much fainter than those of the distributions of the galaxies themselves.

Now the search for the second category of voids, the regions with clear absence of structures, is radically different. This work does not include any cluster correlation computation, so we do not quantify any void of this kind. Such features are too extended for an eventual identification directly from the distribution of galaxies, as no significance levels can be attached. However, much confidence is attributed to these voids since the delimiting structures are themselves highly significant.

Nevertheless, visual inspection of our "positive" detection results clearly reports the presence of some such very extended voids. Firstly, we can report a noticeable void lying "behind" the DN structure (domain [D180], $\alpha=$ 9.0 deg., $\delta=-50.0$ deg., see Fig. 4.): this region remains empty in any domain fainter than magnitude $B_{j}=18.0$. Next, it must be noticed that two magnitude slices, [D185] and [D200], are themselves devoid of significant structures almost throughout the whole map (see Figs. 5 and 9). Such lack of galaxies in the corresponding ranges of magnitudes provides evidence of the presence of voids, simply crossed by the corresponding slices. In this case these two particular slices each cross a very large void: the empty zones are each extended by about $40 \times 40$ degrees!

Since the evidence of these voids comes from "visual impression" and not from the direct analysis of a distribution we do not attribute any label to them and do not describe any characteristics in a table.

\section{The multi-scale database}

This section is the core of the present work. In Tables 12 we summarise all the results discussed above. The structures are present in a basic hierarchical order: first, the very large scales $(\{\mathrm{W}\}$ and $\{\mathrm{V}\})$, then the intermediate scales $(\{\mathrm{S}\}$ and $\{\mathrm{E}\})$, and finally the smaller scales $(\{\mathrm{A}\})$.

In total, we find evidence for 311 distinct structures covering all the different classes. Now, by considering the typical sizes of the structures within the individual categories and comparing the different classes of magnitudes we can compute an approximate estimate for the true dimensions of each feature. For example, consider the redshift values $z=0.05$ to $z=0.1$, i.e. from the foreground to the background domains. A preliminary examination of the redshift surveys compared to our range of magnitudes indicates this to be a reasonable statement. This gives for the dimension of the largest structures detected ( $\{\mathrm{W}\}$-class) values between 20 to $40 \mathrm{~h}^{-1} \mathrm{Mpcs}$. The next class of structures $(\{\mathrm{V}\}$-class $)$ corresponds, on the same basis, to dimensions from 10 to $20 \mathrm{~h}^{-1}$ Mpcs. The intermediate structures and superclusters $(\{\mathrm{E}\}$-class and $\{\mathrm{S}\}$ class) have dimensions of the order of 5 to $10 \mathrm{~h}^{-1}$ Mpcs. The cluster-sized structures $(\{\mathrm{C}\}$-class) have values of the order of $2 \mathrm{~h}^{-1} \mathrm{Mpcs}$, and finally the lower structures $(\{\mathrm{A}\}$ class) have the usual value of about $1 \mathrm{~h}^{-1} \mathrm{Mpc}$.

Of course, the validity of such a computation is entirely related to the knowledge of the true redshift data, for the large quantity of galaxies involved allows us to give a reasonable confidence in the above statements. In all cases we can recapitulate the main hierarchy and the corresponding extension of all the structures detected as follows:

- 6 Ultra-large structures ( $\{\mathrm{W}\}$-class $)$ of 16 to $28 \zeta \cdot \mathrm{h}^{-1}$ Mpcs.

- 19 Very large structures ( $\{\mathrm{V}\}$-class), 19 Superclusters ( $\{\mathrm{S}\}$-class), and 42 Intermediate or Elongated structures (\{E\}-class) of 5 to $18 \zeta \cdot h^{-1}$ Mpcs.

- 91 Cluster-sized structures ( $\{\mathrm{C}\}$-class) of 1.5 to $3 \zeta \cdot \mathrm{h}^{-1}$ Mpcs.

- 134 Compact clusters or Associations (\{A\}-class) of $\sim 1 \zeta \cdot \mathrm{h}^{-1} \mathrm{Mpc}$.

where $\mathrm{h}$ is the usual Hubble constant in units of 100 , and $\zeta$ is the distance module: redshift expressed in units of 0.05 .

Such a complex hierarchy, including in particular very extended structures, suggests departure from the standard Cold Dark Matter picture.

\section{Discussion}

\subsection{The ACO catalogue}

The most immediate application of our results is clearly a comparison with the classical Abell catalogue in its Southern extension, commonly referred to as ACO (Abell et al. 
1989). Table 13 gives the final list of the ACO clusters identified with our own structures. The only criterion for such an identification was the separation of both centroids and the consistency of the respective magnitude ranges. We gave no regard to any correspondence in dimensions, since both definitions of structures come from very different concepts: from a multi-scale point of view structures are not considered with a fixed radius. In some cases, a duplicate exists (an Abell cluster equally matches two structures), in which case we just give the two possibilities.

There are 148 Abell clusters within the region of sky analysed. All of them are identified with at least one of the structures we detect. 19 of these clusters, however, do correspond to structures detected with low significance levels and were not retained in our final list. The remaining 129 Abell clusters are "significant" accordingly to our main procedure, therefore leading to a confidence level of about $87 \%$ for this subsample of the ACO catalogue.

\subsection{Further applications}

Any list of possible further applications of the database as presented in this work should include the following items: - comparison with spectroscopic data: many redshift catalogues are now becoming available, e.g. the recent Edinburgh-Milano Cluster Redshift Survey (Collins et al. 1995), and so a systematic identification with our own structures leads directly to the estimation of the dimensions in real units (Mpc).

- a comparison with X-ray luminosity maps will bring additional information on the dynamics of the structures detected, in particular the correlation which may exist between the X-ray emissions of structures of different classes (sizes, richnesses, etc).

- a reestimate of the $n$-point spatial correlation function is suitable from the use of the respective class of object, since e.g. the two point function is well approximated by the same power law for many classes of objects, from galaxies to superclusters, while its richness dependence is still debated (see Mann et al. 1994 for a detailed discussion). This is also an immediate way to check whether we do evidence significant departure from Gaussian fluctuations.

\section{Conclusion}

We have presented here the topology of a substantial part of the Southern sky, detecting structures from a multi-scale analysis of the two-dimensional distribution ofgalaxies in the COSMOS/UKST Southern Sky Galaxy Catalogue. We detect structures in a wide range of sizes, from Very Large structures of more than 10 degrees in extent (presumably greater than $50 \mathrm{~h}^{-1} \mathrm{Mpc}$ ) to small size clusters. Some remarkable and extended voids are also detected. The algorithm used in this work is based on the multi-scale wavelet transform, and allows us to extract the main individual characteristics such as the position, extent, and significance level of the features detected (both structures and voids). The problem of projection effects was resolved by performing a preliminary analysis on successive magnitude slices and extracting statistics from sets of exact replicas. From this we note an evolutionary effect regarding the classes of magnitude that we attribute to the distinctive morphological behaviour with distance, e.g. the presence of large voids. In total, we present a large and homogeneous database obtained in a fully objective way that includes 25 Ultra-large structures, 61 Superclusters or Very Large structures, and 225 cluster-sized structures.

Acknowledgements. This work has been supported by the E.E.C, from the "Human Capital and Mobility Programme" (grant No. ERBCHBICT930317).

\section{References}

Abell G.O., Corwin H.G.Jr., Olowin R.P., 1989, ApJS 70, 1

Brandenberger R.H., 1989, J. Phys. G. 15, 1

Coles P., Plionis M., 1991, MNRAS 250, 75

Collins C.A., Guzzo. L., Nichol R.C., Lumsden S.L., 1995, MNRAS 274, 1071

Einasto M., Einasto J., Tago E., Dalton G.B., 1994, MNRAS 269,301

Escalera E., Mazure A., 1992, ApJ 388, 23

Escalera E., MacGillivray H.T., 1995, A\&A 298, 1

Gott J.R., et al., 1989, ApJ 340, 625

Ikeuchi S., 1981, Publs. Astr. Soc. Japan 33, 211

MacGillivray H.T., Yentis D.J., 1994, in Astronomy from Wide-Field Imaging, IAU Symposium No. 161. In: MacGillivray H.T. et al. (eds.). Kluwer Academic Publishers, Dordrecht, p. 632

Mann R.G., Peacock J., Heavens A., 1994, MNRAS 263, 798

Messina A., Moscardini L., Lucchin F., Matarrese S., 1990, MNRAS 245, 244

Melott A.N., Cohen A.P., Hamilton A.J.S., Gott J.R., Weinberg D.H., 1989, ApJ 345, 618

Melott A.N., Weinberg D.H., Gott J.R., 1988, ApJ 328, 50

Moscardini L., Matarrese S., Lucchin F., Messina A., 1991, MNRAS 248, 424

Ostriker J.P., Cowie L.L., 1981, ApJ 243, L127

Vilenkin A., 1985, Phys. Rep. 121, 263 


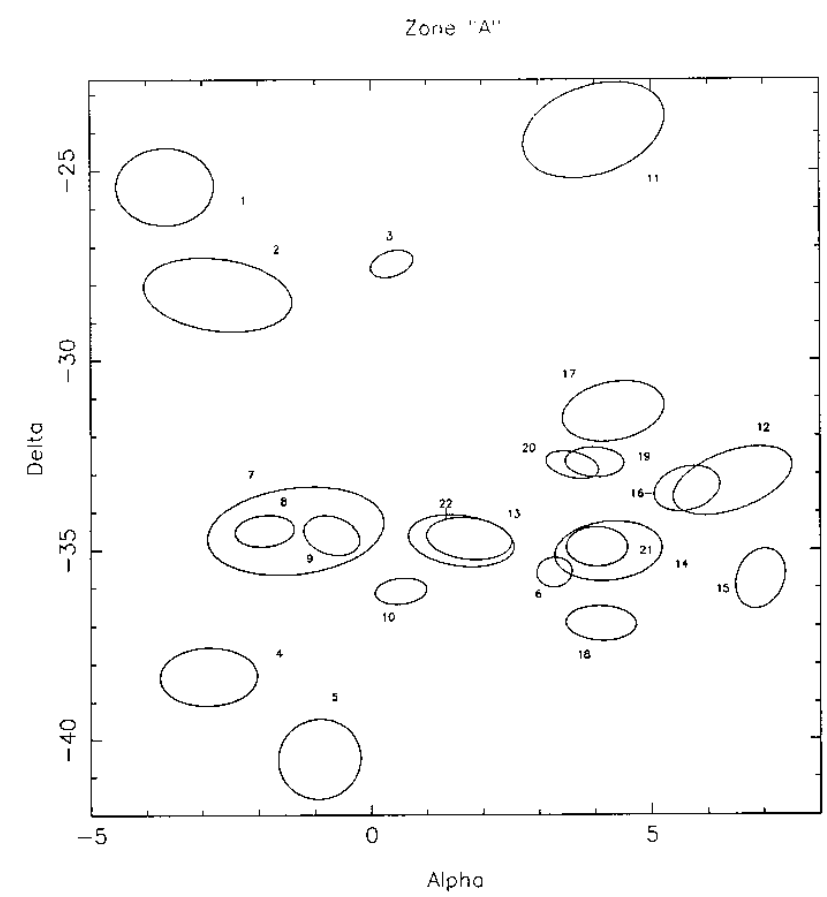

Fig. 13. Representation of the structures detected in zone "A"

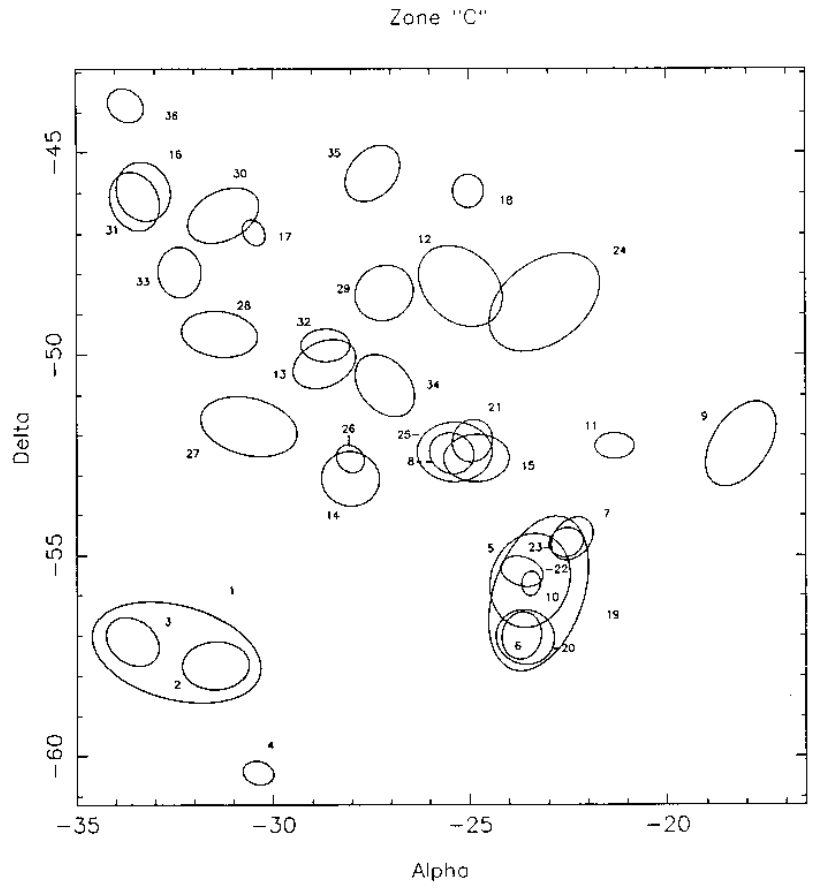

Fig. 15. Representation of the structures detected in zone "C"

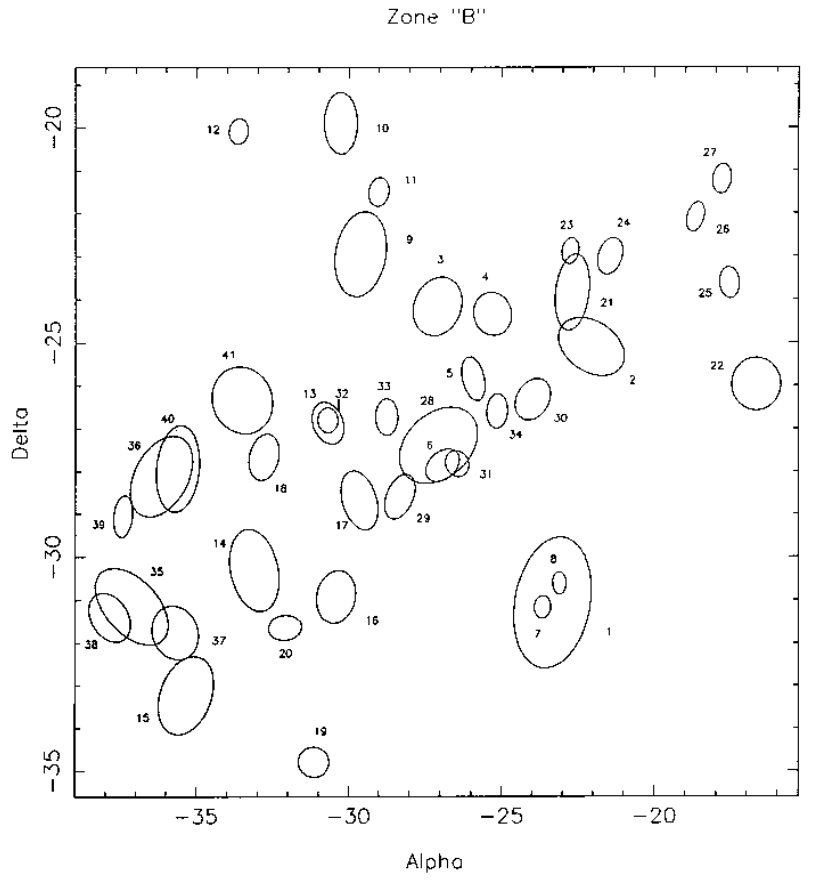

Fig. 14. Representation of the structures detected in zone "B"

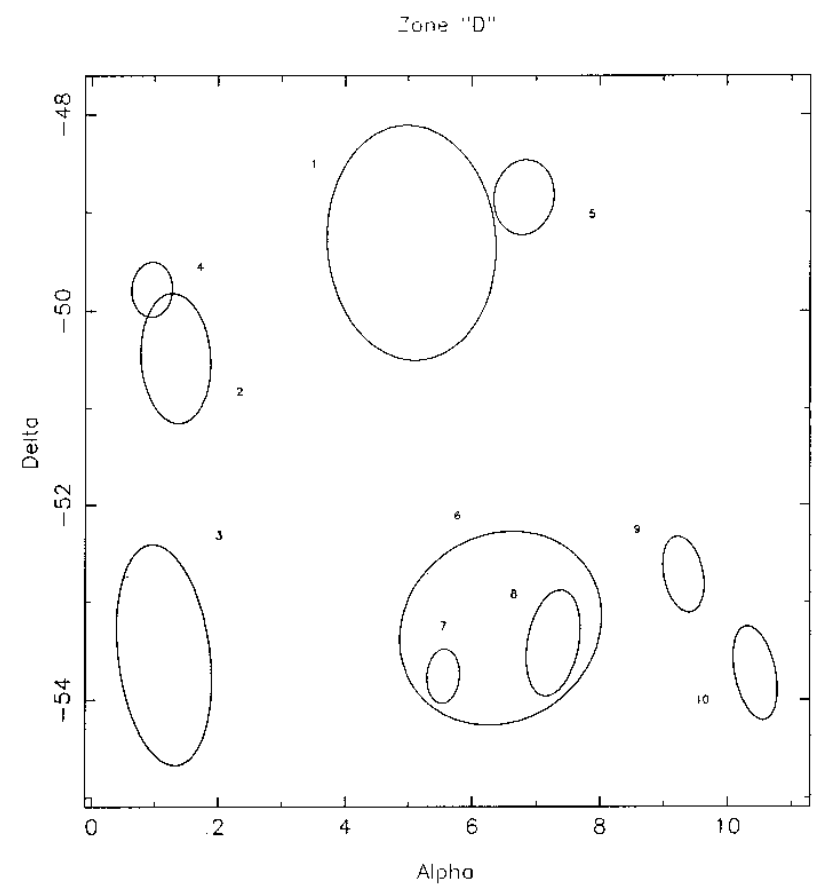

Fig. 16. Representation of the structures detected in zone " $D$ " 

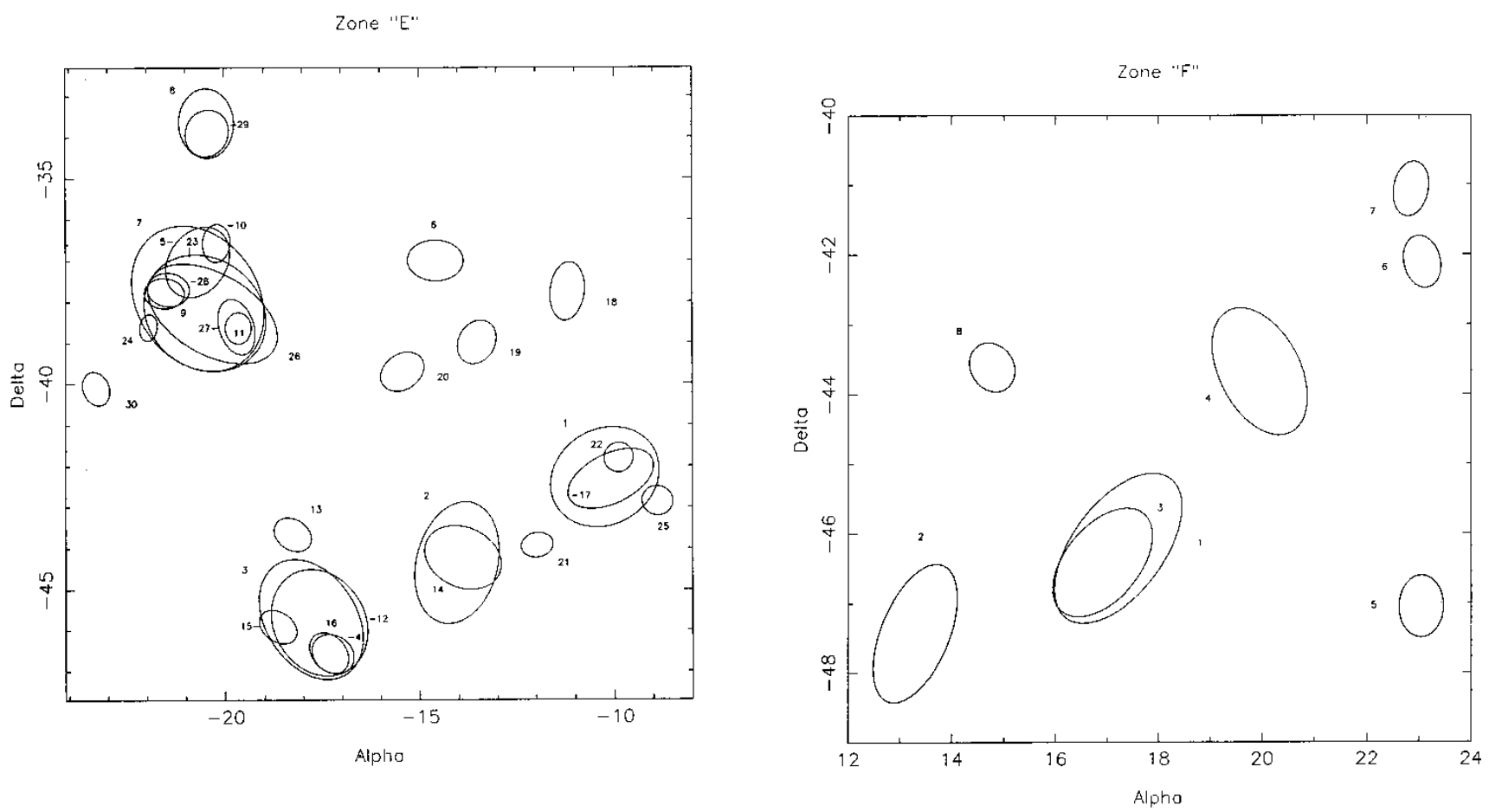

Fig. 17. Representation of the structures detected in zone "E"

Fig. 18. Representation of the structures detected in zone "F"
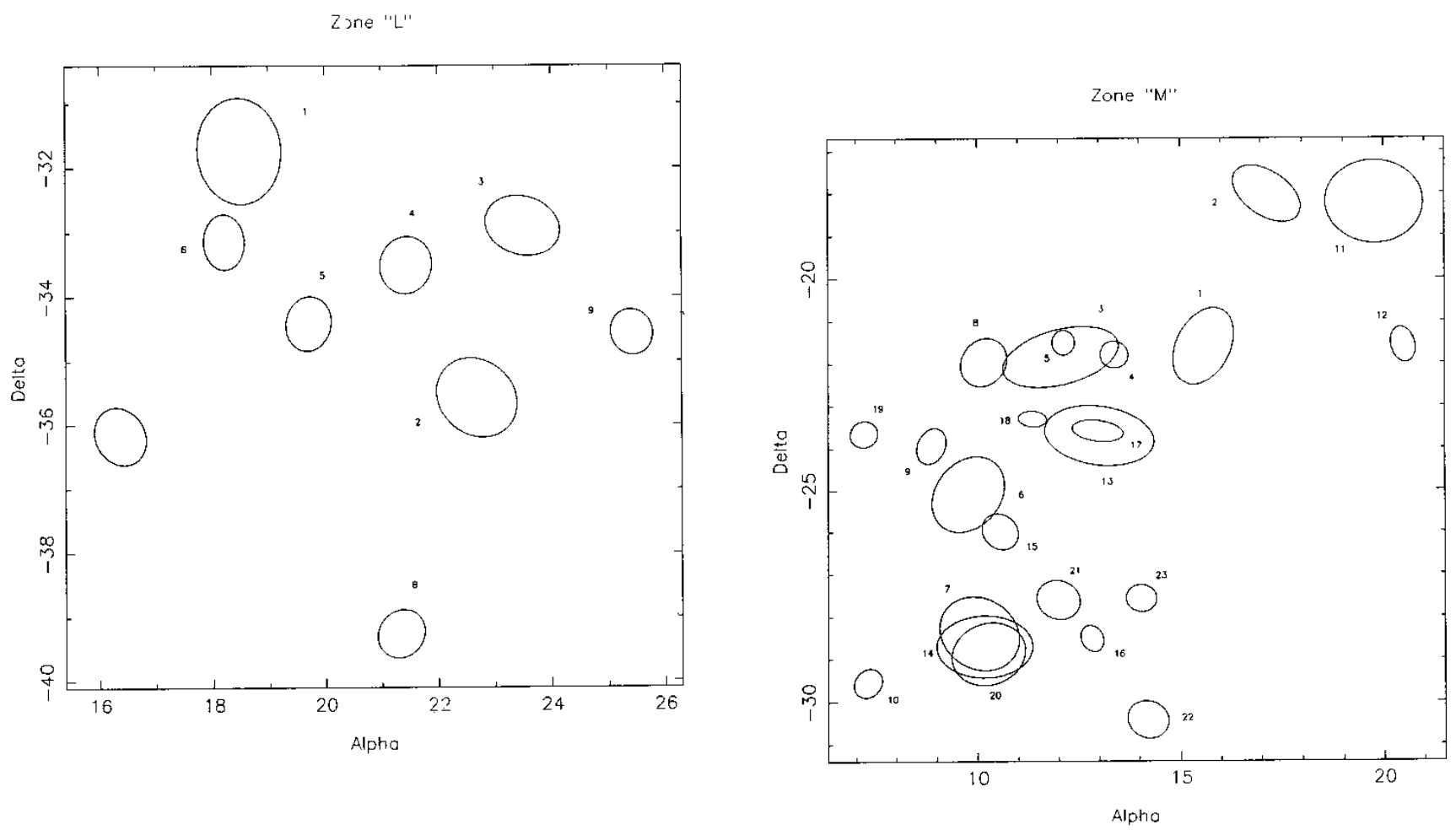

Fig. 19. Representation of the structures detected in zone "L"

Fig. 20. Representation of the structures detected in zone "M" 


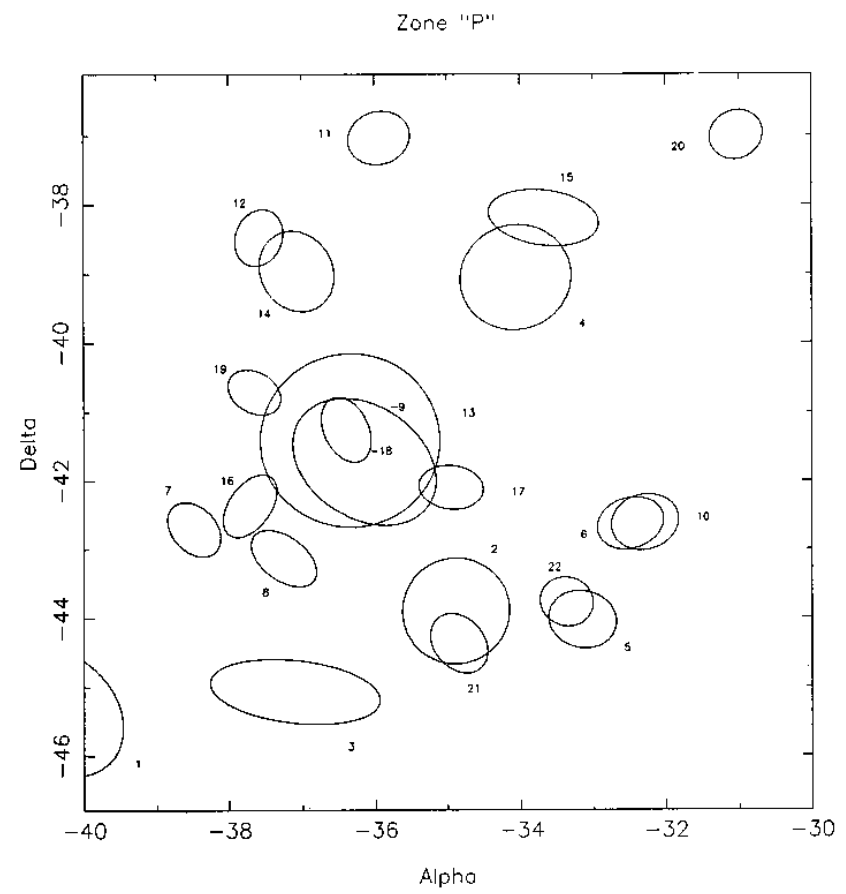

Fig. 21. Representation of the structures detected in zone "P" Fig. 22. Representation of the structures detected in zone "T"

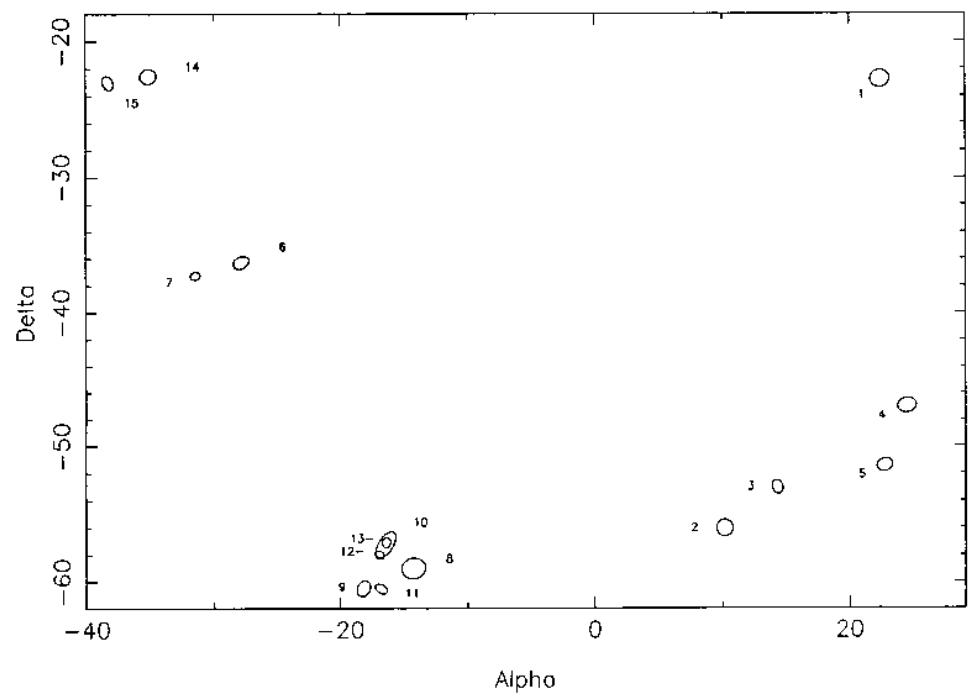

Fig. 23. Representation of the structures detected in zone "V". This image represents the loose structures that are not included in the previous zones. Boundaries are as for the main field (Figs. 1-12) 


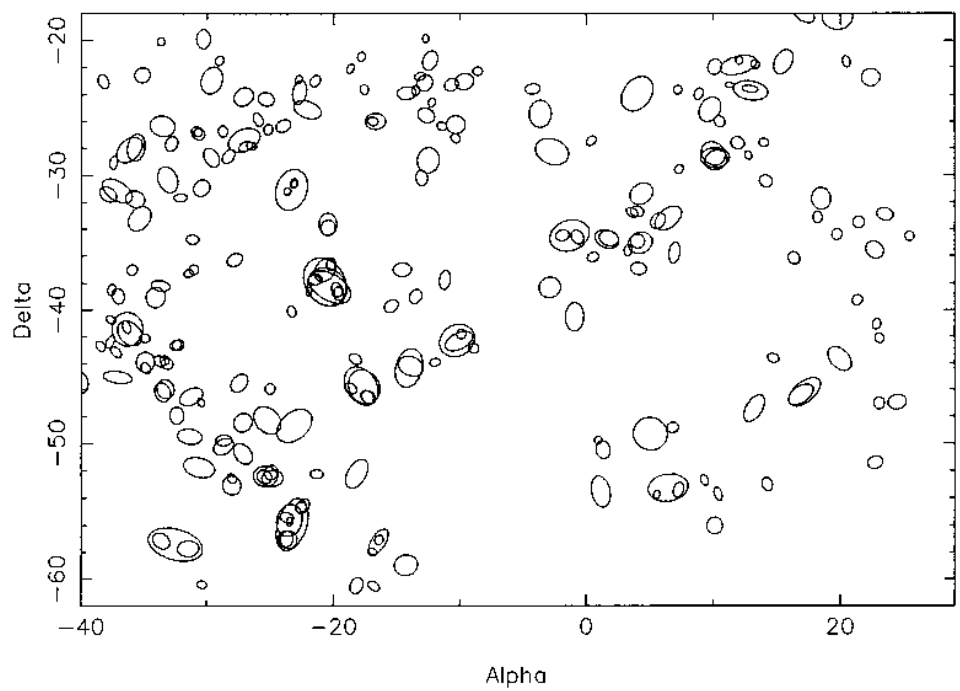

Fig. 24. Representation of the structures, all zones together. Ellipses are not labelled to keep the figure clear. Boundaries are as for the main field (Figs. 1-12), so this gives a representation of the smaller scale detection in the regions of the superclusters. This figure is directly superposable to the ones from the large scales detections, showing at a glance the complex topology of the complete sample analysed

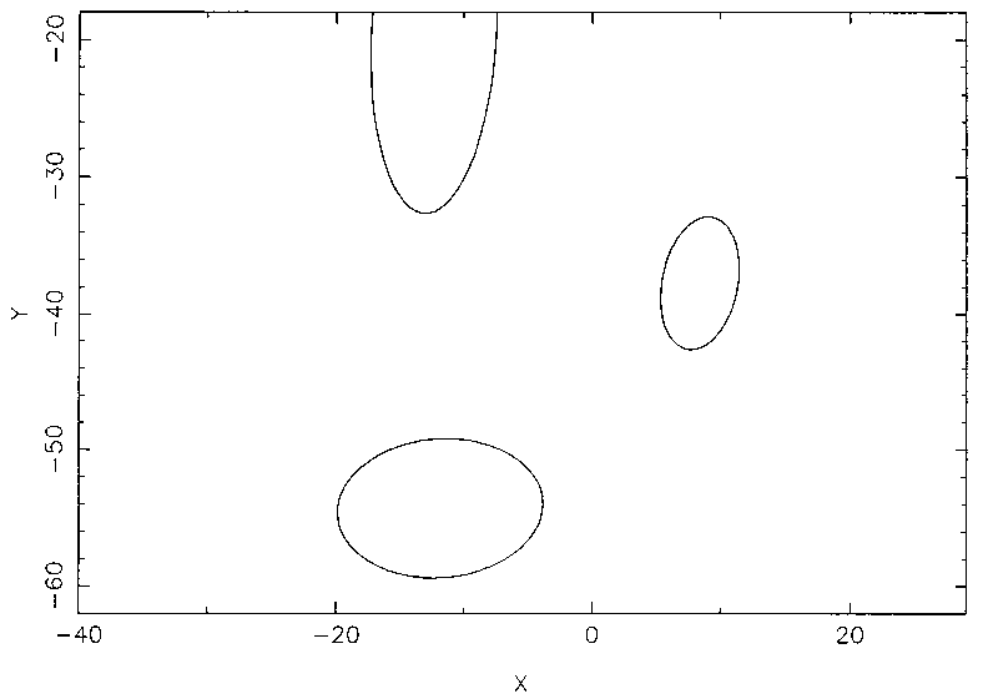

Fig. 25. Representation of the 3 significant voids detected, as listed in Table 11. The upper and the middle one are present in the domains [D085] to [D169] (compare to the Figs. 1-3), while the middle one is exclusive to the domain [D085]. Boundaries are as for the main field (Figs. 1-12, and 24). 